\title{
Modeling Single-Screw Extrusion Processing Parameters and Resulting Extrudate Properties of DDGS-Based Nile Tilapia (Oreochromis niloticus) Feeds
}

\author{
Ferouz Y. Ayadi ${ }^{1}$, Parisa Fallahi ${ }^{1}$, Kurt A. Rosentrater ${ }^{2} \&$ Kasiviswanathan Muthukumarappan ${ }^{1}$ \\ ${ }^{1}$ Department of Agricultural and Biosystems Engineering, South Dakota State University, USA \\ ${ }^{2}$ Department of Agricultural and Biosystems Engineering, Iowa State University, Ames, IA, USA \\ Correspondence: Kurt A. Rosentrater, Department of Agricultural and Biosystems Engineering, Iowa State \\ University, Ames, IA 50011, USA. Tel: 1-515-294-4019. E-mail: karosent@iastate.edu
}

Received: November 22, 2012 Accepted: January 19, $2013 \quad$ Online Published: February 27, 2013

doi:10.5539/jfr.v2n2p11

URL: http://dx.doi.org/10.5539/jfr.v2n2p11

\begin{abstract}
A single-screw laboratory extruder was used to conduct an $\mathrm{L}_{18}\left(2^{2} \times 3^{6}\right)$ Taguchi fractional factorial study of aquafeed processing. The ingredients were based on a formulation for nutritionally-balanced Nile tilapia diets containing distillers dried grains with solubles (DDGS) and soybean meal as the main protein sources, in addition to constant amounts of corn flour, whey, and fishmeal. The effects of three levels of DDGS (20, 30 and $40 \%$ ), soybean meal (30, 40 and 50\%), ingredient moisture content (20, 30 and 40\% db), screw speed (100, 150 and $200 \mathrm{rpm}$ ), die dimension (L/D ratios of 5, 9 and 13$)$, barrel temperature $\left(80-100-100^{\circ} \mathrm{C}, 80-120-120^{\circ} \mathrm{C}\right.$ and $80-140-140^{\circ} \mathrm{C}$ ) and two levels of screw configuration (compression ratios of $2: 1$ and $3: 1$ ) on extrudate physical properties (moisture content, water activity, bulk density, unit density, expansion ratio, pellet durability index, water absorption and solubility indices, water stability, color) and extruder processing parameters (resulting temperatures, die pressure, extruder torque, mass flow rate, apparent viscosity, and specific mechanical energy) were determined. Data from raw materials, processing conditions, and extrudate properties were used to develop surface response curves and equations. However, predominantly low $\mathrm{R}^{2}$ values $(<0.5)$ only permitted linear relationships between some independent parameters and response variables. Regarding main effects, die pressure significantly decreased with higher DDGS levels, moisture content, temperature, lower die L/D, and higher screw compression. Expansion ratio decreased significantly with higher moisture content and lower die L/D. Significant differences in color were caused by changes in DDGS levels and moisture content. In summary, DDGS, moisture content, die dimension, and extrusion conditions had the biggest impact on most of the extrudate physical properties and processing conditions. Different combinations of these independent factors can be used to achieve desired extrudate physical properties and processing conditions.
\end{abstract}

Keywords: alternative protein, aquaculture, extrusion, modeling, physical properties

Abbreviations: DDGS: Distillers dried grains with solubles

\section{Introduction}

Depletion of wild fisheries, combined with rising demands for seafood products for human foods, has led to increased aquaculture production during the last several decades. Depending upon the species and maturity, fish have high dietary protein demands of up to $55 \%$ (NRC, 1993). Fish meal is one of the main protein sources used in aquatic feeds; because of good amino acid balance, high palatability, and growth performance, continually increasing demand for the limited supply of wild fish has steadily increased fish meal prices. For example prices for fish meal for July 2010 were above $\$ 1370$ per ton, compared to \$600 per ton for July 2005 (USDA, 2010). For certain fish species, feed costs alone can represent up to $70 \%$ of the total production cost for an aquaculture operation (Webster \& Lim, 2002; Metts et al., 2007). Protein accounts for the major feed cost. Studies have shown that less expensive alternative protein sources can, at least partially, replace fish meal, satisfy protein demands, and result in good growth performance. These alternatives include various animal and plant sources. Ayadi et al. (2010) provided a comprehensive review of many of these feed ingredients. For instance, meat and bone meal has been used in salmonids feeds (Bureau et al., 2000). Poultry by-product meal has been used in commercial diets for sunshine bass and hybrid striped bass (Rawles et al., 2009; Rawles et al., 2010). Soybean 
meal (SBM) is one of the most studied and widely used plant protein sources in commercial aquatic feeds for many species, such as tilapia, hybrid striped bass, rainbow trout, Atlantic salmon (Salmo salar) and sunshine bass (Morone chrysops $\times$ M. saxatilis) (Steffens, 1994; Thompson et al., 2008; Furuya et al., 2004; Rawles et al., 2009). Distillers dried grains with solubles (DDGS) is another ingredient. It is the major nonfermentable coproduct of fuel ethanol production, and is mostly made from corn grain. Compared to other protein sources, such as SBM, DDGS is very competitive on a cost per unit protein basis, highly palatable to fish (Lim et al., 2009), and does not contain anti-nutritional factors that are present in most pulses. In numerous studies, DDGS has been examined as a potential protein ingredient in fish feed for species such as Nile tilapia, channel catfish, and rainbow trout (Webster et al., 1993; Wu et al., 1996; Cheng \& Hardy, 2004; Lim et al., 2007).

Nile tilapia (Oreochromis niloticus) originated in Africa, and is one of the most important cultured fish species worldwide. Global tilapia production has increased exponentially within the last 30 years. For example, in 1998, 0.7 million metric tonnes ( $\mathrm{t}$ ) of Nile tilapia were produced, compared to 2.3 million $\mathrm{t}$ in 2008, of which Asia has become the major producer (FAO, 2010). For the US market, tilapia production increased from 15,521 $\mathrm{t}$ in 1998 to $81,130 \mathrm{t}$ in 2008 (FAO, 2010). It is the fifth most popular seafood consumed in the US (ATA, 2010). Tilapia has relatively fast growth, undemanding feed conditions, and physical hardiness (Fitzsimmons, 2006). Even though Nile tilapia has been classified as herbivorous, it has been reported that Nile Tilapia can also feed on insects, algae, and potentially other fish (Njiru et al., 2004). Protein requirements depend on maturity; they can be up to $45 \%$ for Nile tilapia fry (El-Sayed \& Teshima, 1992; Hafedh, 1999), whereas bigger fish can require down to $30 \%$ protein or less (Hafedh, 1999; Bahnasawy, 2009).

Dietary components are only one aspect of fish feeding, however. The other is feed production. High quality aquatic feeds are commonly produced by extrusion processing, which can produce floating or sinking feeds and improve nutrient digestibility (Pezzato, 1999). Extrusion processing has become very popular in the feed and food industries due to high versatility, productivity, and product quality. Previous research by our group has focused on several processing aspects of DDGS-based feeds. Single-screw and twin-screw extrusion have been used to produce feeds for tilapia, channel catfish, yellow perch, and rainbow trout. The effects of various levels of DDGS inclusion, ingredient moisture content, protein content screw speed, barrel temperature, and die dimension, on resulting extruder processing conditions and extrudate properties have been examined (Chevanan et al., 2007a, 2007b, 2007c, 2008, 2009, 2010; Kannadhason et al., 2009a, 2009b, 2010; Rosentrater et al., 2009a, 2009b; Ayadi et al., 2011a, 2011b, 2011c). Additionally, we have used these extruded feeds in feeding trials to test their efficacy (Schaeffer et al., 2009, 2010). Most of these studies, however, were empirical and deterministic in nature. Follow-up modeling studies on extrusion can help to predict output parameters (e.g. extrudate properties) based on extruder processing settings and/or formulations of the raw blends.

Extrusion cooking involves many complex processes that can be difficult to control due to interactions between mass, energy, and momentum transfer phenomena. Physicochemical changes impact extrudate properties, and can be difficult to predict (Wang et al., 2001). Some research has been conducted on modeling of extrusion processes and resulting product quality. For example, Meng et al. (2010) used second-order polynomial regression to model twin-screw extruder system parameters (feed moisture content, screw speed, and barrel temperature) and physical properties of chickpea flour-based snacks. A similar modeling study was accomplished by Ding et al. (2005) for rice-based expanded snacks. Chevanan et al. (2007c) developed neural network and regression models of single-screw extrusion of aquaculture feeds containing DDGS; data collected from different trials were combined to predict extrudate properties and extrusion processing parameters based on die dimensions, ingredient moisture content, barrel temperature, and screw speed. Wang et al. (2001) modeled twin-screw extrusion to control extrudate quality attributes. Multiple regression models were developed by Ganjal et al. (2004) to relate the radial expansion of extrudates to die nozzle dimensions and back pressure at the die for acetylated starch in a twin-screw extruder. Ali et al. (1996) developed a regression model to study the effects of temperature and screw speed on the radial, axial, and overall expansion, as well as bulk density of extruded corn grits in a single-screw extruder.

In this study, the goal was to model the effects of various levels of DDGS, soybean meal, ingredient moisture content, screw speed, screw compression ratio, die dimension, and barrel temperature on resulting extrudate physical properties (e.g., moisture content, water activity, bulk density, unit density, expansion ratio, pellet durability index, water absorption and water solubility indices, water stability, and color) and on resulting extruder processing conditions (barrel temperature, die pressure, extruder torque, mass flow rate, apparent viscosity, and specific mechanical energy). 


\section{Materials and Methods}

\subsection{Feed Blend Preparation}

DDGS was provided by Dakota Ethanol, LLC (Wentworth, SD) and soybean meal was obtained from Dakotaland Feeds, LLC (Huron, SD). Low temperature menhaden fish meal was purchased from Consumers Supply Distributing Co. (Huron, SD); corn flour was from Cargill Dry Corn Ingredients, Inc. (Paris, IL); dried whey was from Midor Ltd. (Elroy, WI).

Six ingredient blends (Table 1) were adjusted to a target protein content of $\sim 30 \% \mathrm{db}$, a target fat content of $\sim 17 \%$ $\mathrm{db}$, and three different moisture contents of 20,30 , and $40 \% \mathrm{db}$. With increasing DDGS levels $(20,30,40 \% \mathrm{db})$, and thus decreasing soybean meal levels $(50,40,30 \%)$, but constant levels of fish meal (approximately), corn flour, and whey, these ingredients were used to prepare nutritionally-balanced diets for Nile tilapia (Schaeffer et al., 2010; Chevanan et al., 2007b). DDGS and soybean meal were ground with a laboratory mill (Model 4, Thomas Scientific, Swedesboro, NJ) to a flour with an average particle size of approximately 500 micrometer $(\mu \mathrm{m})$. The whey was sieved in manually (Sieve No. 14, ASTM E-11, Daigger, Vernon Hills, IL) to prevent coagulation within the blends. The components were then blended in a rotary mixer for $10 \mathrm{~min}$ (Kushlan Products, Inc., Goldendale, WA). After all ingredients were thoroughly combined, each blend was adjusted to the desired moisture content of 20,30, and $40 \%$, respectively, by adding adequate amounts of water, and then thoroughly mixed using a laboratory-scale mixer (Professional 6, KitchenAid, St. Joseph, MI).

Table 1. Ingredient components (for each diet) used in the study

\begin{tabular}{llll}
\hline & \multicolumn{3}{l}{ Dry weight of ingredients $(\mathrm{g} / \mathrm{kg})$} \\
\hline Diet1 & Diet2 & Diet3 \\
\hline Soybean meal & 20 & 30 & 40 \\
Corn flour & 50 & 40 & 30 \\
Whey & 15 & 15 & 15 \\
Fishmeal & 5 & 5 & 5 \\
\hline TOTAL & 10 & 10 & 10 \\
\hline
\end{tabular}

\subsection{Experimental Design and Extrusion Processing}

$\mathrm{A} \mathrm{L}_{18}\left(2^{2} \times 3^{6}\right)$ Taguchi fractional factorial design (Table 2) was used for the study. The treatment combinations consisted of 18 uniquetrials, which consisted of different combinations of 2 levels of screw compression ratio (2:1, 3:1), 2 levels of fishmeal (9.99\% and 9.98\%), 3 levels of DDGS (20, 30 and 40\%), 3 levels of soybean meal (30, 40 and 50\%), 3 levels of raw blend moisture content $(20,30$ and $40 \% \mathrm{db}), 3$ levels of screw speed ( 100 , 150 and $200 \mathrm{rpm}$ ), 3 levels of die dimension (L/D ratios of 5, 9 and 13), and 3 levels of barrel temperature profile (80-100-100, 80-120-120 and 80-140-140 $\left.{ }^{\circ} \mathrm{C}\right)$.

A single-screw extruder (Model PL 2000, Brabender Plasti-Corder, South Hackensack, NJ), with a barrel length of $317.5 \mathrm{~mm}$, was used to extrude each blend. Three different dies were used, with length to diameter (L/D) ratios of 5, 9 and 13. The center of the die assembly was conical, and tapered from an initial diameter of $6.0 \mathrm{~mm}$ to an exit diameter of 2.0, 3.2 or $6.0 \mathrm{~mm}$, respectively, at the discharge opening. A $7.5 \mathrm{HP}(5.5 \mathrm{~kW})$ motor was connected to the extruder drive shaft. During extrusion, the screw speed was adjusted to 100, 150 and $200 \mathrm{rpm}$, respectively. For all runs, the temperature of the feed zone was controlled and maintained at $80^{\circ} \mathrm{C}$, that of the transition zone at 100,120 , or $140^{\circ} \mathrm{C}$, respectively, and that of the die zone at 100,120 , or $140^{\circ} \mathrm{C}$, respectively. The raw blends were manually funneled into the extruder in constant quantities to avoid jamming at the opening of the barrel and to provide a continuous feed. All processing data were collected every $60 \mathrm{~s}$, and the average of eight $(n=8)$ recordings were used for statistical analyses, except for mass flow rate where three samples $(n=3)$ were used.

\subsection{Raw Ingredient Properties}

Each raw blend was analyzed for moisture content, water activity, and color (Hunter L, a, b values). Methods used will be discussed subsequently. 
Table 2. Experimental design used in the study

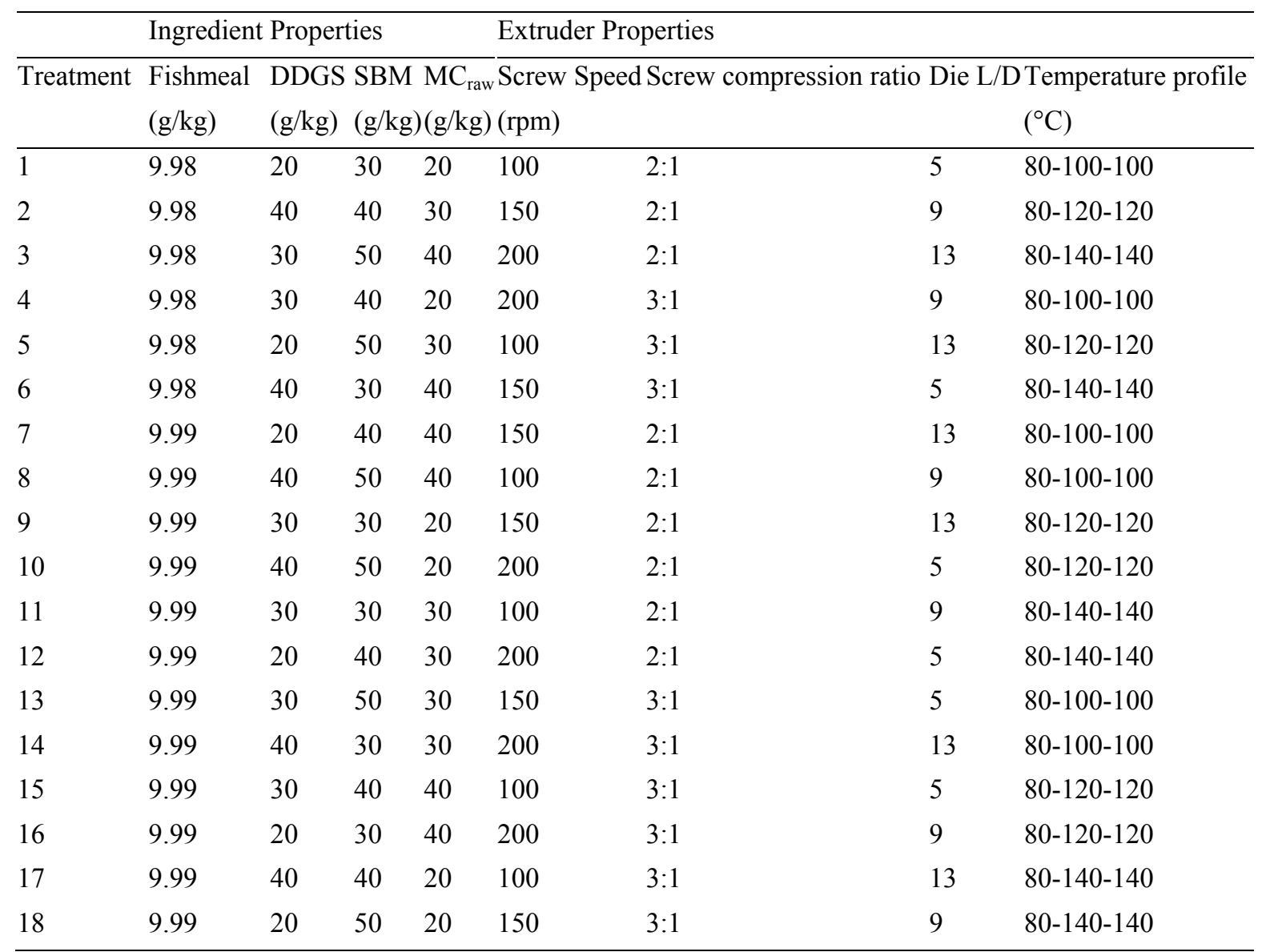

* The experimental design consisted of an $\mathrm{L}_{18}$ Taguchi fractional factorial design with 18 total treatment combinations. $\mathrm{MC}_{\text {raw }}$ is raw blend moisture content, die $\mathrm{L} / \mathrm{D}$ is length to diameter ratio of the die, $\mathrm{SBM}$ is soybean meal

\subsection{Extrusion Processing Parameters}

\subsubsection{Temperature Profile, Die Pressure and Torque}

The absolute pressure at the die zone and the actual temperature profile at the feed, metering, and die zones were simultaneously monitored every minute for eight $(\mathrm{n}=8)$ recordings using a combined thermocouple/pressure transducer (GP50, New York Ltd., Grand Island, NY). Likewise, the net torque exerted on the screw drive shaft was recorded with a torque transducer (Measurement Specialists, Huntsville, AL) at a sensing range of 0-390 N.m every minute for eight $(\mathrm{n}=8)$ recordings.

\subsubsection{Mass Flow Rate (MFR)}

Extrudate samples exiting the die were collected at $30 \mathrm{~s}$ intervals, dried, and weighed using an electronic balance (PB 5001, Mettler Toledo, Switzerland) to quantify the mass flow rate.

\subsubsection{Apparent Viscosity $\left(\eta_{\text {app }}\right)$}

The extruder was approximated as a coaxial cylinder-shaped viscometer, where the screw and barrel were considered as an inner and an outer cylinder, respectively (Rogers, 1970; Lu et al., 1992; Rosentrater et al., 2005; Chevanan et al., 2007a). The apparent viscosity of the dough was calculated using:

$$
\eta_{a p p}=\left(\frac{\mathrm{C}_{\mathrm{ss}}}{\mathrm{C}_{\mathrm{sr}}}\right) \times\left(\frac{\tau}{\omega}\right)
$$

where $\eta_{\text {app }}$ is the apparent viscosity of the dough $(\mathrm{Pa} \bullet \mathrm{s}), \tau$ is the net torque exerted on the screw shaft $(\mathrm{N} \bullet \mathrm{m}), \omega$ is the screw speed (rpm), Css is an empirical correction factor for the shear rate which relates to the screw configuration, and Csr is an empirical correction factor for the shear rate which relates to the barrel size, where: 


$$
\begin{gathered}
C_{s s}=\frac{1}{2 \pi \cdot L_{s} \cdot r_{c o r r}{ }^{2}} \\
C_{s r}=\frac{2 r_{b}^{2}}{r_{b}^{2}-r_{\text {corr }}^{2}} \\
r_{\text {Corr }}=\sqrt{\left(r_{e f f_{1}}^{2}+r_{e f f_{1}} \cdot r_{e f f_{2}}+r_{e f f_{2}}^{2}\right) / 3}
\end{gathered}
$$

where $r_{\text {corr }}$ is the radius correction factor due to the frustum geometry of the screw $(m), r_{b}$ is the barrel radius $(m), L_{S}$ is the screw length in the axial direction $(\mathrm{m})$, and $\mathrm{r}_{\text {eff }}$ is the effective radius of the screw obtained from the sum of the screw root and half of the flight height $(\mathrm{m})$. Specific values for these parameters have been discussed elsewhere (Rosentrater et al., 2005).

\subsubsection{Specific Mechanical Energy (SME)}

Specific mechanical energy (J/g) was calculated using equation (5), following Harper (1981):

$$
S M E=\frac{\tau \cdot \omega \cdot 60}{m_{\text {feed }}}
$$

where $\tau$ is the net torque exerted on the screw shaft (N.m), $\omega$ is the screw speed (rpm), and $m_{\text {feed }}$ is the mass flow rate of the input dry feed $(\mathrm{g} / \mathrm{min})$, calculated using the following equation:

$$
m_{\text {feed }}=M F R \cdot\left(\frac{1-M C_{f}}{1-M C_{e}}\right)
$$

where $\mathrm{MC}_{\mathrm{f}}$ is the moisture content of the raw feed blend $(\% \mathrm{wb})$ and $\mathrm{MC}_{\mathrm{e}}$ is the moisture content of the extrudate at the die $(\% \mathrm{wb})$.

\subsubsection{Extrudate Physical Properties}

After the prepared blends were cooked in the extruder and dried for $72 \mathrm{~h}$ at room temperature $\left(25 \pm 1^{\circ} \mathrm{C}\right)$, they were then analyzed for moisture content $(\% \mathrm{db})$, water activity, bulk density $\left(\mathrm{kg} / \mathrm{m}^{3}\right)$, unit density $\left(\mathrm{kg} / \mathrm{m}^{3}\right)$, expansion ratio, pellet durability index (\%), water absorption and water solubility indices (\%), water stability ( $\mathrm{min})$, and color. For all treatment runs, three samples $(\mathrm{n}=3)$ were used to determine the physical properties.

\subsubsection{Moisture Content (MC)}

According to AACC method 44-19 (2000), the moisture content of the raw material and extrudate samples for each blend were determined using a laboratory oven (Thelco Precision, Jovan, Wincester, VA) at $135^{\circ} \mathrm{C}$ for $2 \mathrm{~h}$.

\subsubsection{Water Activity $\left(\mathrm{a}_{\mathrm{w}}\right)$}

Water activity was measured for the raw material and extrudate samples from each treatment with a water activity meter (aw Sprint TH-500, Novasina, Pfäffikon, Switzerland). The sample bowl was filled with each sample and then placed into the measuring chamber of the pre-calibrated instrument.

\subsubsection{Bulk Density (BD)}

Bulk density (BD) was determined as the ratio of the mass of extrudates in a given bulk volume. A standard bushel tester (Seedburo Equipment Company, Chicago, IL) was used following the method described by USDA (1999).

\subsubsection{Unit Density (UD)}

The extrudates were cut to a length of $25.4 \mathrm{~mm}$, weighed on an analytical balance (Adventurer ${ }^{\mathrm{TM}}$, Item No. AR 1140, Ohaus Corp. Pine Brook, NJ), and then measured with a digital caliper (Model No. CD-6' 'C, Mitutoyo Corp., Tokyo, Japan) to determine their diameter. According to Rosentrater et al. (2005) the unit density (UD, $\left.\mathrm{kg} / \mathrm{m}^{3}\right)$ was calculated as the ratio of the mass $\mathrm{m}(\mathrm{kg})$ to the volume $\mathrm{V}\left(\mathrm{m}^{3}\right)$ of each measured and weighed extrudate sample, assuming a cylindrical shape for each extrudate:

$$
\mathrm{UD}=m / V
$$

\subsubsection{Expansion Ratio (ER)}

The diameter of the extrudates was measured with a digital caliper (Digimatic caliper, Model No: CD-6" C, Mitutoyo Corp., Tokyo, Japan), and then the ratio at that diameter to the diameter of the die nozzle (2.0, 3.2, or 6.0 $\mathrm{mm}$ ) was used to quantify the radial expansion ratio. 


\subsubsection{Pellet Durability Index (PDI)}

Pellet durability index was determined following Method S269.4 (ASAE, 2004). Approximately $100 \mathrm{~g}$ of extrudates from each blend were manually sieved (ASTM E-11, Daigger, Vernon Hills, IL) for about $10 \mathrm{~s}$ to remove initial fines, and then tumbled in a pellet durability tester (Model PDT-110, Seedburo Equipment Company, Chicago, IL) for $10 \mathrm{~min}$. Afterwards, the samples were again hand sieved for about $10 \mathrm{~s}$, and then weighed on an electronic balance (Explorer Pro, Model. EP4102, Ohaus, Pine Brook, NJ). PDI was calculated as:

$$
\text { PDI }(\%)=(M a / M b) \times 100
$$

where $\mathrm{M}_{\mathrm{a}}$ is the mass $(\mathrm{g})$ after tumbling and $\mathrm{M}_{\mathrm{b}}$ is the sample mass $(\mathrm{g})$ before tumbling.

\subsubsection{Water Absorption and Water Solubility Index}

Water absorption index (WAI) and water solubility index (WSI) were measured according to the method of Anderson et al. (1969) and Jones et al. (2000). Extrudate sample of each treatment combination were ground with a cyclone mill (Cyclone Sample Mill, Model 3010-830, UDY Corporation, Fort Collins, CO) to an average particle size of about $500 \mu \mathrm{m}$. Approximately $2.5 \mathrm{~g}$ of the extrudate powder was suspended in $30 \mathrm{~mL}$ of water in a tarred 50 $\mathrm{mL}$ centrifuge tube. The tube was placed in a laboratory oven (Thelco Precision, Jovan, Wincester, VA) at $30^{\circ} \mathrm{C}$ and stirred periodically every $10 \mathrm{~min}$ for $30 \mathrm{~min}$. Afterwards, the water-extrudate suspension was centrifuged for $15 \mathrm{~min}$ at $3000 \mathrm{rpm}$ in a laboratory-scale centrifuge (Durafuge 100, Precision, Winchester, VA). The supernatant was decanted into tarred aluminum dishes and dried for $2 \mathrm{~h}$ at $135^{\circ} \mathrm{C}$ in the laboratory oven. The ratio of the remaining gel mass in the centrifuge tube to the original sample mass (approximately $2.5 \mathrm{~g}$ ) was used to determine the water absorption index:

$$
W A I=W g / W S
$$

where $\mathrm{W}_{\mathrm{g}}$ is gel weight $(\mathrm{g})$ and $\mathrm{W}_{\mathrm{s}}$ is the original sample weight $(\mathrm{g})$.

WSI was calculated as the ratio of the dry solids (remaining from evaporation of the supernatant from the WAI test) to the original sample mass, following AACC Method 44-19 (2000).

$$
W S I(\%)=(W d s / W s) \times 100
$$

where $\mathrm{W}_{\mathrm{ds}}$ is the dry weight of the supernatant $(\mathrm{g})$ and $\mathrm{W}_{\mathrm{s}}$ is original weight of the sample $(\mathrm{g})$.

\subsubsection{Water Stability}

Water stability is defined as the amount of time that it takes for an extrudate to begin to break apart after it has been placed in water. For extrudates of each blend, a 1-g sample was placed in $200 \mathrm{~mL}$ of distilled water and gently stirred using a magnet stirrer (PMC No. 524C, Barnstead International, Dubuque, IA) until the extrudates began to visibly dissolve, and the time was then recorded.

\subsubsection{Color}

A spectrophotometer (LabScan XE, HunterLab, Reston, VA) was used to determine color, where L quantified brightness/darkness, a redness/greenness, and b yellowness/blueness of the samples.

\subsection{Statistical Analysis}

Each blend was extruded once. For each treatment combination, three replicates $(n=3)$ were determined for all physical properties. All collected data were analyzed with Microsoft Excel v.2007 and SAS v.9 (SAS Institute, Cary, NC). The Proc GLM (general linear models) procedure was used to identify the main effects (i.e., individual effects due to each independent variable) and the treatment (simultaneous) combination effects using a Type I error rate $(\alpha)$ of 0.05 . Then, post-hoc LSD tests were used to determine where the specific differences occurred. TableCurve 3D v.4.0.01 (SYSTAT Software, Inc., San Joes, CA) was also used for response surface modeling.

\section{Results}

\subsection{Extrusion Processing Parameters}

\subsubsection{Die Pressure}

The barrel of the extruder essentially acts as a pressure cooker, where steam and pressure are released at the die opening (Harper, 1981). The design of the die can impact pressure release as well as result in additional pressure. Die pressure and temperature highly affect expansion and mass flow of extrudate. At lower temperatures and lower pressures, less water evaporates which results in less expansion. Hence, moisture content and screw speed are important factors that affect die pressure and extrudate expansion as well. 
With changes in screw speed, no significant differences were detected for the die pressure for the main effects (Table 4). This may be related to the high standard deviations. Generally, all standard deviations for the recorded parameters for processing conditions (SME, torque, viscosity) were relatively high. The highest value for die pressure was recorded at $1603.00 \mathrm{MPa}$ (Run 4), while the lowest value was at $42.50 \mathrm{MPa}$ (Run 15) (Table 5). As expected, the die pressure decreased with higher moisture content. This is in agreement with other extrusion studies (Lin et al., 2002; Meng et al., 2010; Singh et al., 2007). At high moisture water can act as a lubricant, and will reduce friction of the extruded dough, which in turn decreases die pressure (Lin et al., 2002). This is reflected in the values for the $a_{w}$, which increased with higher blend moisture content. The data for raw blend $a_{w} v a r i e d$ between $0.62-0.66,0.74-0.77$, and $0.78-0.80$ for 20,30 , and $40 \%$ moisture content, respectively (Table 3 ). Raising the processing temperature from 100 to $140^{\circ} \mathrm{C}$ resulted in a significant decrease in die pressure of $43.2 \%$ (Table 4). Similar results were reported by other investigators (Fletcher et al., 1985; Kirby et al., 1988; Singh et al., 2007). Furthermore, the die pressure showed a significant decrease with a larger die diameter. The die pressure dropped by $46.0 \%$ by decreasing the die L/D from 13 to 5 (Table 4). This is in agreement with other observations (Sokhey et al., 1997), and was expected due to an increasing die area, and thus less resistance to flow. Increasing the DDGS level from 20 to $40 \%$ yielded a drop in pressure by $31.4 \%$. Likewise, a similar trend was observed by Chevanan et al. (2010). Changes due to increasing DDGS, MC, temperature, and die diameter resulted in significantly lower die pressure values. Examining the treatment combination effects (Table 5) reveals that many treatments were significantly different from each other, which resulted from simultaneous changes of the combined independent variables.

Table 3. Physical properties of the raw feed blends*

\begin{tabular}{|c|c|c|c|c|c|c|c|c|c|c|c|c|c|c|c|c|c|c|}
\hline \multirow[b]{2}{*}{ Property } & \multicolumn{18}{|c|}{ Treatment } \\
\hline & 1 & 2 & 3 & 4 & 5 & 6 & 7 & 8 & 9 & 10 & 11 & 12 & 13 & 14 & 15 & 16 & 17 & 18 \\
\hline $\begin{array}{l}a_{w} \\
(-)\end{array}$ & $\begin{array}{l}0.64 \mathrm{~g} \\
(0.00)\end{array}$ & $\begin{array}{l}0.74 \mathrm{e} \\
(0.00)\end{array}$ & $\begin{array}{l}0.80 a \\
(0.00)\end{array}$ & $\begin{array}{l}0.66 f \\
(0.00)\end{array}$ & $\begin{array}{l}0.77 d \\
(0.00)\end{array}$ & $\begin{array}{l}0.78 \mathrm{c} \\
(0.00)\end{array}$ & $\begin{array}{c}0.80 \mathrm{ab} \\
(0.00)\end{array}$ & $\begin{array}{c}0.80 \mathrm{ab} \\
(0.00)\end{array}$ & $\begin{array}{l}0.62 \mathrm{~h} \\
(0.00)\end{array}$ & $\begin{array}{l}0.62 \mathrm{~h} \\
(0.00)\end{array}$ & $\begin{array}{l}0.74 \mathrm{e} \\
(0.00)\end{array}$ & $\begin{array}{l}0.74 \mathrm{e} \\
(0.01)\end{array}$ & $\begin{array}{l}0.74 \mathrm{e} \\
(0.00)\end{array}$ & $\begin{array}{l}0.74 \mathrm{e} \\
(0.00)\end{array}$ & $\begin{array}{l}0.79 b \\
(0.00)\end{array}$ & $\begin{array}{c}0.80 \mathrm{ab} \\
(0.00)\end{array}$ & $\begin{array}{l}0.62 \mathrm{~h} \\
(0.00)\end{array}$ & $\begin{array}{l}0.62 \mathrm{~h} \\
(0.02)\end{array}$ \\
\hline $\begin{array}{l}\mathrm{L} \\
(-)\end{array}$ & $\begin{array}{c}62.43 a \\
(0.82)\end{array}$ & $\begin{array}{c}55.05 \mathrm{de} \\
(1.37)\end{array}$ & $\begin{array}{c}54.04 \mathrm{ef} \\
(1.33)\end{array}$ & $\begin{array}{c}56.76 \mathrm{~cd} \\
(0.93)\end{array}$ & $\begin{array}{c}51.57 \mathrm{~g} \\
(1.11)\end{array}$ & $\begin{array}{c}53.18 \mathrm{fg} \\
(0.84)\end{array}$ & $\begin{array}{c}53.99 \mathrm{ef} \\
(0.61)\end{array}$ & $\begin{array}{c}52.94 \mathrm{fg} \\
(0.46)\end{array}$ & $\begin{array}{c}59.16 \mathrm{~b} \\
(2.08)\end{array}$ & $\begin{array}{c}58.23 \mathrm{bc} \\
(1.19)\end{array}$ & $\begin{array}{c}57.30 \mathrm{c} \\
(0.55)\end{array}$ & $\begin{array}{c}59.11 \mathrm{~b} \\
(0.92)\end{array}$ & $\begin{array}{c}57.30 \mathrm{c} \\
(0.55)\end{array}$ & $\begin{array}{c}55.05 \mathrm{de} \\
(1.37)\end{array}$ & $\begin{array}{c}54.04 \mathrm{ef} \\
(1.33)\end{array}$ & $\begin{array}{c}53.99 \text { ef } \\
(0.61)\end{array}$ & $\begin{array}{c}58.23 \mathrm{bc} \\
(1.19)\end{array}$ & $\begin{array}{r}62.43 a \\
(0.82)\end{array}$ \\
\hline $\begin{array}{l}a \\
(-)\end{array}$ & $\begin{array}{l}3.63 g \\
(0.18)\end{array}$ & $\begin{array}{l}5.63 \mathrm{~b} \\
(0.10)\end{array}$ & $\begin{array}{l}5.57 \mathrm{~b} \\
(0.35)\end{array}$ & $\begin{array}{l}4.54 \mathrm{ef} \\
(0.31)\end{array}$ & $\begin{array}{c}5.38 \mathrm{bc} \\
(0.33)\end{array}$ & $\begin{array}{l}6.24 a \\
(0.18)\end{array}$ & $\begin{array}{l}4.98 \mathrm{~d} \\
(0.21)\end{array}$ & $\begin{array}{l}6.45 a \\
(0.19)\end{array}$ & $\begin{array}{l}4.34 f \\
(0.16)\end{array}$ & $\begin{array}{l}5.13 c d \\
(0.07)\end{array}$ & $\begin{array}{r}4.86 \mathrm{de} \\
(0.13)\end{array}$ & $\begin{array}{l}4.38 f \\
(0.07)\end{array}$ & $\begin{array}{r}4.86 \mathrm{de} \\
(0.13)\end{array}$ & $\begin{array}{l}5.63 b \\
(0.10)\end{array}$ & $\begin{array}{l}5.57 \mathrm{~b} \\
(0.35)\end{array}$ & $\begin{array}{l}4.98 d \\
(0.21)\end{array}$ & $\begin{array}{c}5.13 \mathrm{~cd} \\
(0.07)\end{array}$ & $\begin{array}{l}3.63 \mathrm{~g} \\
(0.18)\end{array}$ \\
\hline $\begin{array}{l}\mathrm{b} \\
(-)\end{array}$ & $\begin{array}{c}18.59 \mathrm{~g} \\
(0.47)\end{array}$ & $\begin{array}{c}20.71 \mathrm{abc} \\
(0.45)\end{array}$ & $\begin{array}{c}20.49 \mathrm{bcd} \\
(0.72)\end{array}$ & $\begin{array}{c}19.15 \text { efg } \\
(0.91)\end{array}$ & $\begin{array}{c}18.82 f g \\
(0.97)\end{array}$ & $\begin{array}{c}21.28 \mathrm{ab} \\
(0.42)\end{array}$ & $\begin{array}{c}19.74 \mathrm{de} \\
(0.33)\end{array}$ & $\begin{array}{r}21.43 a \\
(0.32)\end{array}$ & $\begin{array}{c}19.24 \mathrm{efg} \\
(0.59)\end{array}$ & $\begin{array}{c}20.01 \mathrm{cde} \\
(0.36)\end{array}$ & $\begin{array}{c}19.91 \mathrm{cde} \\
(0.32)\end{array}$ & $\begin{array}{c}19.63 \mathrm{def} \\
(0.22)\end{array}$ & $\begin{array}{c}\text { 19.91cde } \\
(0.32)\end{array}$ & $\begin{array}{c}20.71 \mathrm{abc} \\
(0.45)\end{array}$ & $\begin{array}{c}20.49 \mathrm{bcd} \\
(0.72)\end{array}$ & $\begin{array}{c}19.74 \mathrm{de} \\
(0.33)\end{array}$ & $\begin{array}{c}20.01 \mathrm{cde} \\
(0.36)\end{array}$ & $\begin{array}{l}18.59 \mathrm{~g} \\
(0.47)\end{array}$ \\
\hline
\end{tabular}

* Means followed by similar letters for a given dependent variable are not significantly different among treatments at $\mathrm{P}<0.05$, LSD. Values in parentheses are standard deviation. $\mathrm{a}_{\mathrm{w}}$ is water activity, $\mathrm{L}$ is brightness, $\mathrm{a}$ is redness/greenness, $\mathrm{b}$ is yellowness/blueness.

\subsubsection{Torque}

Torque quantifies the force that is required to rotate the extruder screw. Thus, it is affected by the viscosity of the dough, moisture content, temperature, and screw speed (Akdogan, 1996). High torque requires more energy and can lead to wear of the extruder. Optimal torque values can save energy and reduce stress on the equipment.

Regarding the main treatment effects (Table 4), no clear pattern of changes in torque could be observed for several independent variables, which again might be related to the high standard deviations. The highest torque value was recorded for run 13 at 51.73 N.m, whereas the lowest was recorded for run 8 at 8.26 N.m (Table 5). Some significant differences in torque were detected with interactive changes across the treatment combinations (Table 5). The torque decreased with higher levels of DDGS, and with higher moisture content. Conversely, torque increased with higher levels of SBM. Changes in screw speed yielded no significant differences in torque for the main effects, which was related to the high standard deviations. But the dough did exhibit shear thinning behavior: the apparent viscosity decreased significantly by $42.2 \%$ when increasing the screw speed from 100 to $200 \mathrm{rpm}$ (Table 4). The different screw configurations also had a significant effect on torque. This was as expected, due to the changes in flight height of the screw which increased compression and thus torque for the 2:1 compression ratio screw versus the 3:1 compression ratio. And, as die L/D increased, the torque increased due to greater resistance to flow, which resulted in a higher pressure.

\subsubsection{Mass Flow Rate}

A common way to examine the productivity of an extruder is to measure its output. Previous studies have shown that the amount of extrudate produced per unit time is impacted by screw speed, die geometry, shear rate, diet formulation (such as DDGS level), moisture content, and the viscosity of the dough melt (Chevanan et al., 2008; Kannadhason et al., 2010). In this study, except for screw speed, none of the independent variables had significant effects on MFR for the main effects, which again was related to the high standard deviations observed in the data. 
For the main effects, with increasing screw speed from 100 to $150 \mathrm{rpm}$, MFR increased significantly by $109.8 \%$ (Table 4). The highest MFR was detected for run 3 at $225.60 \mathrm{~g} / \mathrm{min}$, while the lowest was for run 8 at $67.13 \mathrm{~g} / \mathrm{min}$ (Table 5). Treatment combination effects were also studied and response surface generated for MFR, SS, and DDGS level (Figure 1). As shown in Figure 1, the higher screw speed and lower levels of DDGS substitution resulted in decreased flow rate. Overall, changing in DDGS levels in diet did not have considerable impact on extrudate output.

Table 4. Main effects of DDGS, SBM, moisture content of raw material, screw speed, screw compression ratio, die $\mathrm{L} / \mathrm{D}$, and extruder temperature profile on raw blends, measured processing properties, and extrudate physical properties*

\begin{tabular}{|c|c|c|c|c|c|c|c|c|c|}
\hline \multirow[b]{2}{*}{ Variable } & \multicolumn{4}{|c|}{ Raw Materials } & \multicolumn{5}{|c|}{ Processing conditions } \\
\hline & $a_{\text {wRaw }}$ & $\mathrm{L}_{\text {Raw }}$ & $a_{\text {Raw }}$ & $b_{\text {Raw }}$ & $\begin{array}{c}\text { P at Die } \\
\text { (Mpa) }\end{array}$ & $\begin{array}{c}\text { Torque } \\
(\mathrm{N} \mathrm{m})\end{array}$ & $\begin{array}{c}\text { MFR } \\
\text { (g/min) }\end{array}$ & $\begin{array}{c}\eta_{\mathrm{app}} \\
\text { (Pa s) }\end{array}$ & $\begin{array}{l}\text { SME } \\
(\mathrm{J} / \mathrm{g})\end{array}$ \\
\hline \multicolumn{10}{|c|}{ DDGS (\% db) } \\
\hline 20 & $\begin{array}{l}0.73 a \\
(0.07)\end{array}$ & $\begin{array}{c}57.25 a \\
(4.48)\end{array}$ & $\begin{array}{l}4.50 c \\
(0.72)\end{array}$ & $\begin{array}{c}19.19 \mathrm{c} \\
(0.70)\end{array}$ & $\begin{array}{l}932.04 a \\
(484.21)\end{array}$ & $\begin{array}{l}29.14 a \\
(11.98)\end{array}$ & $\begin{array}{c}115.32 a \\
(31.43)\end{array}$ & $\begin{array}{c}1880.83 a \\
(1094.42)\end{array}$ & $\begin{array}{l}860.90 a \\
(367.52)\end{array}$ \\
\hline 30 & $\begin{array}{l}0.73 a \\
(0.07)\end{array}$ & $\begin{array}{c}56.43 a \\
(2.17)\end{array}$ & $\begin{array}{l}4.96 \mathrm{~b} \\
(0.53)\end{array}$ & $\begin{array}{c}19.86 \mathrm{~b} \\
(0.76)\end{array}$ & $\begin{array}{c}790.56 a b \\
(580.72)\end{array}$ & $\begin{array}{r}30.41 a \\
(17.13)\end{array}$ & $\begin{array}{c}129.67 a \\
(56.00)\end{array}$ & $\begin{array}{c}1637.52 a \\
(783.61)\end{array}$ & $\begin{array}{c}716.52 \mathrm{ab} \\
(423.89)\end{array}$ \\
\hline 40 & $\begin{array}{l}0.72 a \\
(0.08)\end{array}$ & $\begin{array}{c}55.45 a \\
(2.38)\end{array}$ & $\begin{array}{l}5.70 c \\
(0.53)\end{array}$ & $\begin{array}{c}20.69 a \\
(0.65)\end{array}$ & $\begin{array}{l}639.02 \mathrm{~b} \\
(302.58)\end{array}$ & $\begin{array}{l}18.43 b \\
(11.10)\end{array}$ & $\begin{array}{c}111.58 a \\
(51.14)\end{array}$ & $\begin{array}{l}1067.25 b \\
(648.75)\end{array}$ & $\begin{array}{l}513.44 b \\
(210.87)\end{array}$ \\
\hline \multicolumn{10}{|c|}{$\operatorname{SBM}(\% \mathrm{db})$} \\
\hline 30 & $\begin{array}{l}0.72 a \\
(0.07)\end{array}$ & $\begin{array}{c}56.85 a \\
(3.44)\end{array}$ & $\begin{array}{l}4.95 a \\
(0.88)\end{array}$ & $\begin{array}{c}19.91 a \\
(0.98)\end{array}$ & $\begin{array}{l}713.02 a \\
(523.26)\end{array}$ & $\begin{array}{l}22.08 b \\
(10.16)\end{array}$ & $\begin{array}{c}102.92 a \\
(34.69)\end{array}$ & $\begin{array}{c}1405.04 b \\
(898.54)\end{array}$ & $\begin{array}{l}606.10 a \\
(220.73)\end{array}$ \\
\hline 40 & $\begin{array}{l}0.73 a \\
(0.07)\end{array}$ & $\begin{array}{c}56.20 \mathrm{a} \\
(2.24)\end{array}$ & $\begin{array}{l}5.04 a \\
(0.52)\end{array}$ & $\begin{array}{c}19.96 a \\
(0.71)\end{array}$ & $\begin{array}{l}787.58 a \\
(576.56)\end{array}$ & $\begin{array}{l}24.54 b \\
(13.36)\end{array}$ & $\begin{array}{c}132.61 a \\
(49.13)\end{array}$ & $\begin{array}{c}1365.37 \mathrm{~b} \\
(624.69)\end{array}$ & $\begin{array}{l}673.92 a \\
(360.37)\end{array}$ \\
\hline 50 & $\begin{array}{l}0.72 a \\
(0.08)\end{array}$ & $\begin{array}{c}56.09 a \\
(3.86)\end{array}$ & $\begin{array}{l}5.17 a \\
(0.89)\end{array}$ & $\begin{array}{c}19.88 a \\
(1.10)\end{array}$ & $\begin{array}{l}861.02 a \\
(299.51)\end{array}$ & $\begin{array}{l}31.36 a \\
(17.89)\end{array}$ & $\begin{array}{c}121.04 a \\
(53.30)\end{array}$ & $\begin{array}{l}1815.18 a \\
(1124.53)\end{array}$ & $\begin{array}{l}810.84 a \\
(471.29)\end{array}$ \\
\hline \multicolumn{10}{|c|}{$M C_{\text {Raw }}(\% d b)$} \\
\hline 20 & $\begin{array}{l}0.63 c \\
(0.02)\end{array}$ & $\begin{array}{c}59.54 a \\
(2.46)\end{array}$ & $\begin{array}{l}4.40 c \\
(0.65)\end{array}$ & $\begin{array}{c}19.27 \mathrm{c} \\
(0.76)\end{array}$ & $\begin{array}{c}1220.75 a \\
(346.90)\end{array}$ & $\begin{array}{l}28.17 a \\
(10.49)\end{array}$ & $\begin{array}{c}106.14 a \\
(35.13)\end{array}$ & $\begin{array}{c}1686.17 a \\
(721.03)\end{array}$ & $\begin{array}{l}624.98 b \\
(186.87)\end{array}$ \\
\hline 30 & $\begin{array}{l}0.75 b \\
(0.01)\end{array}$ & $\begin{array}{c}55.90 \mathrm{~b} \\
(2.61)\end{array}$ & $\begin{array}{l}5.12 b \\
(0.49)\end{array}$ & $\begin{array}{c}19.95 b \\
(0.80)\end{array}$ & $\begin{array}{l}602.00 \mathrm{~b} \\
(312.52)\end{array}$ & $\begin{array}{r}32.29 a \\
(16.94)\end{array}$ & $\begin{array}{c}133.67 a \\
(44.99)\end{array}$ & $\begin{array}{c}1948.87 a \\
(1125.72)\end{array}$ & $\begin{array}{l}929.56 a \\
(505.40)\end{array}$ \\
\hline 40 & $\begin{array}{l}0.79 a \\
(0.01)\end{array}$ & $\begin{array}{c}53.70 \mathrm{c} \\
(0.91)\end{array}$ & $\begin{array}{l}5.63 a \\
(0.62)\end{array}$ & $\begin{array}{c}20.53 a \\
(0.80)\end{array}$ & $\begin{array}{l}538.88 \mathrm{~b} \\
(446.25)\end{array}$ & $\begin{array}{l}17.52 b \\
(11.55)\end{array}$ & $\begin{array}{c}116.76 a \\
(57.15)\end{array}$ & $\begin{array}{l}950.55 b \\
(504.72)\end{array}$ & $\begin{array}{l}536.33 b \\
(208.11)\end{array}$ \\
\hline \multicolumn{10}{|l|}{ SS (rpm) } \\
\hline 100 & - & - & - & - & $\begin{array}{l}718.88 a \\
(474.17)\end{array}$ & $\begin{array}{l}23.18 a \\
(14.08)\end{array}$ & $\begin{array}{c}76.31 \mathrm{c} \\
(7.68)\end{array}$ & $\begin{array}{c}1922.54 a \\
(1129.17)\end{array}$ & $\begin{array}{l}612.02 a \\
(344.64)\end{array}$ \\
\hline 150 & - & - & - & - & $\begin{array}{l}884.60 a \\
(481.08)\end{array}$ & $\begin{array}{l}27.97 a \\
(15.72)\end{array}$ & $\begin{array}{c}120.18 b \\
(41.49)\end{array}$ & $\begin{array}{l}1551.38 b \\
(812.55)\end{array}$ & $\begin{array}{l}710.81 a \\
(397.06)\end{array}$ \\
\hline 200 & - & - & - & - & $\begin{array}{l}758.15 a \\
(485.30)\end{array}$ & $\begin{array}{l}26.83 a \\
(13.81)\end{array}$ & $\begin{array}{c}160.08 a \\
(37.87)\end{array}$ & $\begin{array}{c}1111.67 \mathrm{c} \\
(563.07)\end{array}$ & $\begin{array}{l}768.04 a \\
(366.22)\end{array}$ \\
\hline \multicolumn{10}{|c|}{ Screw comp. } \\
\hline $2: 1$ & - & - & - & - & $\begin{array}{l}950.83 a \\
(521.87)\end{array}$ & $\begin{array}{l}32.05 a \\
(14.26)\end{array}$ & $\begin{array}{c}135.01 a \\
(66.32)\end{array}$ & $\begin{array}{c}1964.79 a \\
(1085.34)\end{array}$ & $\begin{array}{l}769.13 a \\
(269.57)\end{array}$ \\
\hline $3: 1$ & - & - & - & - & $\begin{array}{l}705.40 \mathrm{~b} \\
(441.37)\end{array}$ & $\begin{array}{l}22.97 b \\
(13.88)\end{array}$ & $\begin{array}{c}110.78 a \\
(32.09)\end{array}$ & $\begin{array}{c}1310.41 \mathrm{~b} \\
(744.16)\end{array}$ & $\begin{array}{l}660.87 a \\
(408.05)\end{array}$ \\
\hline \multicolumn{10}{|l|}{ Die L/D } \\
\hline 5 & - & - & - & - & $\begin{array}{l}544.81 \mathrm{c} \\
(508.60)\end{array}$ & $\begin{array}{l}22.06 b \\
(17.97)\end{array}$ & $\begin{array}{c}103.08 a \\
(34.12)\end{array}$ & $\begin{array}{l}1345.70 \mathrm{~b} \\
(1115.82)\end{array}$ & $\begin{array}{l}729.19 a \\
(531.36)\end{array}$ \\
\hline 9 & - & - & - & - & $\begin{array}{l}807.42 b \\
(466.37)\end{array}$ & $\begin{array}{c}25.43 \mathrm{ab} \\
(13.41)\end{array}$ & $\begin{array}{c}127.83 a \\
(47.60)\end{array}$ & $\begin{array}{c}1397.26 \mathrm{~b} \\
(654.04)\end{array}$ & $\begin{array}{l}601.21 a \\
(223.36)\end{array}$ \\
\hline 13 & - & - & - & - & $\begin{array}{c}1009.40 a \\
(346.56)\end{array}$ & $\begin{array}{r}30.50 a \\
(10.44)\end{array}$ & $\begin{array}{c}125.66 a \\
(55.72)\end{array}$ & $\begin{array}{c}1842.63 a \\
(873.81)\end{array}$ & $\begin{array}{l}760.47 a \\
(276.47)\end{array}$ \\
\hline \multicolumn{10}{|l|}{$\mathrm{T}\left({ }^{\circ} \mathrm{C}\right)$} \\
\hline 100 & - & - & - & - & $\begin{array}{c}1002.50 a \\
(463.65)\end{array}$ & $\begin{array}{r}31.77 a \\
(15.90)\end{array}$ & $\begin{array}{c}121.83 a \\
(40.82)\end{array}$ & $\begin{array}{c}1774.88 a \\
(860.92)\end{array}$ & $\begin{array}{l}796.78 a \\
(399.40)\end{array}$ \\
\hline 120 & - & - & - & - & $\begin{array}{l}789.63 \mathrm{~b} \\
(486.22)\end{array}$ & $\begin{array}{l}22.90 \mathrm{~b} \\
(13.93)\end{array}$ & $\begin{array}{c}111.63 \mathrm{a} \\
(44.18)\end{array}$ & $\begin{array}{c}1431.02 \mathrm{ab} \\
(1105.86)\end{array}$ & $\begin{array}{l}627.75 a \\
(326.42)\end{array}$ \\
\hline 140 & - & - & - & - & $\begin{array}{l}569.50 c \\
(399.99)\end{array}$ & $\begin{array}{l}23.31 b \\
(12.28)\end{array}$ & $\begin{array}{c}123.10 a \\
(56.84)\end{array}$ & $\begin{array}{c}1379.70 \mathrm{~b} \\
(728.32)\end{array}$ & $\begin{array}{l}666.34 a \\
(375.58)\end{array}$ \\
\hline
\end{tabular}




\begin{tabular}{|c|c|c|c|c|c|c|c|c|c|c|c|c|}
\hline \multirow[b]{2}{*}{ Variable } & \multicolumn{12}{|c|}{ Extrudate Properties } \\
\hline & $\begin{array}{l}M C_{E x t} \\
(\% d b)\end{array}$ & $a_{w E x t}$ & $\begin{array}{c}\text { BD } \\
\left(\mathrm{kg} / \mathrm{m}^{3}\right)\end{array}$ & $\begin{array}{c}\text { UD } \\
\left(\mathrm{kg} / \mathrm{m}^{3}\right)\end{array}$ & ER & $\begin{array}{l}\text { PDI } \\
(\%)\end{array}$ & WAI & WSI & $\begin{array}{l}\text { WS } \\
(\min )\end{array}$ & $\mathrm{L}_{\mathrm{Ext}}$ & $a_{E x t}$ & $b_{\text {Ext }}$ \\
\hline \multicolumn{13}{|l|}{ DDGS (\% db) } \\
\hline 20 & $\begin{array}{l}9.74 a \\
(1.82)\end{array}$ & $\begin{array}{l}0.41 a \\
(0.04)\end{array}$ & $\begin{array}{c}217.52 \mathrm{a} \\
(27.80)\end{array}$ & $\begin{array}{l}9.74 a \\
(1.82)\end{array}$ & $\begin{array}{l}0.90 a \\
(0.08)\end{array}$ & $\begin{array}{l}94.00 a \\
(4.04)\end{array}$ & $\begin{array}{l}3.37 a \\
(0.26)\end{array}$ & $\begin{array}{c}19.20 a \\
(0.96)\end{array}$ & $\begin{array}{c}20.73 a \\
(7.76)\end{array}$ & $\begin{array}{l}44.09 a \\
(5.05)\end{array}$ & $\begin{array}{l}3.94 b \\
(0.49)\end{array}$ & $\begin{array}{c}12.76 \mathrm{~b} \\
(2.27)\end{array}$ \\
\hline 30 & $\begin{array}{l}8.88 b \\
(0.85)\end{array}$ & $\begin{array}{l}0.41 a \\
(0.04)\end{array}$ & $\begin{array}{c}218.29 a \\
(29.38)\end{array}$ & $\begin{array}{l}8.88 b \\
(0.85)\end{array}$ & $\begin{array}{c}0.8 a \\
(0.18)\end{array}$ & $\begin{array}{c}94.94 a \\
(4.71)\end{array}$ & $\begin{array}{c}3.24 a b \\
(0.35)\end{array}$ & $\begin{array}{l}19.61 a \\
(1.60)\end{array}$ & $\begin{array}{l}20.39 a \\
(10.19)\end{array}$ & $\begin{array}{c}42.27 a \\
(4.31)\end{array}$ & $\begin{array}{l}4.77 a \\
(0.73)\end{array}$ & $\begin{array}{c}14.55 a \\
(1.94)\end{array}$ \\
\hline 40 & $\begin{array}{l}9.00 \mathrm{ab} \\
(0.83)\end{array}$ & $\begin{array}{l}0.39 a \\
(0.04)\end{array}$ & $\begin{array}{c}221.61 a \\
(18.46)\end{array}$ & $\begin{array}{l}9.00 \mathrm{ab} \\
(0.83)\end{array}$ & $\begin{array}{l}0.89 a \\
(0.16)\end{array}$ & $\begin{array}{c}94.85 a \\
(4.36)\end{array}$ & $\begin{array}{l}3.05 b \\
(0.31)\end{array}$ & $\begin{array}{l}18.20 \mathrm{~b} \\
(0.79)\end{array}$ & $\begin{array}{c}22.22 a \\
(6.33)\end{array}$ & $\begin{array}{l}41.91 a \\
(3.62)\end{array}$ & $\begin{array}{l}5.17 a \\
(1.01)\end{array}$ & $\begin{array}{c}14.43 a \\
(1.83)\end{array}$ \\
\hline \multicolumn{13}{|l|}{ SBM (\% db) } \\
\hline 30 & $\begin{array}{l}9.31 \mathrm{a} \\
(1.57)\end{array}$ & $\begin{array}{l}0.41 a \\
(0.04)\end{array}$ & $\begin{array}{c}214.33 a \\
(16.14)\end{array}$ & $\begin{array}{l}9.31 a \\
(1.57)\end{array}$ & $\begin{array}{l}0.86 b \\
(0.16)\end{array}$ & $\begin{array}{c}94.53 a \\
(4.73)\end{array}$ & $\begin{array}{l}3.28 a \\
(0.39)\end{array}$ & $\begin{array}{l}19.50 a \\
(1.77)\end{array}$ & $\begin{array}{c}21.22 \mathrm{ab} \\
(9.94)\end{array}$ & $\begin{array}{c}42.22 a \\
(3.65)\end{array}$ & $\begin{array}{c}4.68 \mathrm{ab} \\
(1.01)\end{array}$ & $\begin{array}{c}13.93 a \\
(2.52)\end{array}$ \\
\hline 40 & $\begin{array}{l}9.22 a \\
(1.44)\end{array}$ & $\begin{array}{l}0.40 a \\
(0.04)\end{array}$ & $\begin{array}{c}228.11 a \\
(31.62)\end{array}$ & $\begin{array}{l}9.22 a \\
(1.44)\end{array}$ & $\begin{array}{l}0.86 b \\
(0.17)\end{array}$ & $\begin{array}{c}95.20 a \\
(3.07)\end{array}$ & $\begin{array}{l}3.35 a \\
(0.25)\end{array}$ & $\begin{array}{c}18.55 b \\
(0.92)\end{array}$ & $\begin{array}{l}17.34 b \\
(6.74)\end{array}$ & $\begin{array}{c}42.33 a \\
(3.90)\end{array}$ & $\begin{array}{l}4.94 a \\
(1.06)\end{array}$ & $\begin{array}{c}14.32 \mathrm{a} \\
(2.24)\end{array}$ \\
\hline MO & $\begin{array}{l}9.10 a \\
(0.78)\end{array}$ & $\begin{array}{l}0.40 a \\
(0.04)\end{array}$ & $\begin{array}{c}214.97 a \\
(24.46)\end{array}$ & $\begin{array}{l}9.10 a \\
(0.78)\end{array}$ & $\begin{array}{l}0.95 a \\
(0.07)\end{array}$ & $\begin{array}{c}94.07 a \\
(5.06)\end{array}$ & $\begin{array}{l}3.02 b \\
(0.26)\end{array}$ & $\begin{array}{c}18.95 a b \\
(0.88)\end{array}$ & $\begin{array}{c}24.78 a \\
(5.71)\end{array}$ & $\begin{array}{c}43.71 a \\
(5.48)\end{array}$ & $\begin{array}{l}4.26 \mathrm{~b} \\
(0.49)\end{array}$ & $\begin{array}{c}13.50 a \\
(1.64)\end{array}$ \\
\hline \multicolumn{13}{|l|}{$M C_{\text {Raw }}(\% \mathrm{db})$} \\
\hline 20 & $\begin{array}{l}8.79 \mathrm{~b} \\
(1.23)\end{array}$ & $\begin{array}{l}0.41 a \\
(0.05)\end{array}$ & $\begin{array}{c}231.54 a \\
(26.40)\end{array}$ & $\begin{array}{l}8.79 b \\
(1.23)\end{array}$ & $\begin{array}{l}0.99 a \\
(0.06)\end{array}$ & $\begin{array}{l}91.96 \mathrm{~b} \\
(5.00)\end{array}$ & $\begin{array}{l}3.17 a \\
(0.27)\end{array}$ & $\begin{array}{c}18.85 a b \\
(0.97)\end{array}$ & $\begin{array}{l}17.44 b \\
(6.67)\end{array}$ & $\begin{array}{c}45.39 a \\
(3.25)\end{array}$ & $\begin{array}{l}5.06 a \\
(1.09)\end{array}$ & $\begin{array}{c}15.54 a \\
(1.16)\end{array}$ \\
\hline 30 & $\begin{array}{l}8.89 b \\
(0.99)\end{array}$ & $\begin{array}{l}0.40 a \\
(0.04)\end{array}$ & $\begin{array}{c}228.40 a \\
(16.86)\end{array}$ & $\begin{array}{l}8.89 b \\
(0.99)\end{array}$ & $\begin{array}{l}0.92 b \\
(0.09)\end{array}$ & $\begin{array}{c}94.34 b \\
(3.66)\end{array}$ & $\begin{array}{l}3.31 a \\
(0.38)\end{array}$ & $\begin{array}{c}19.51 a \\
(1.73)\end{array}$ & $\begin{array}{l}18.42 \mathrm{~b} \\
(7.91)\end{array}$ & $\begin{array}{l}44.62 a \\
(3.53)\end{array}$ & $\begin{array}{c}4.61 a b \\
(0.70)\end{array}$ & $\begin{array}{c}14.47 \mathrm{~b} \\
(1.42)\end{array}$ \\
\hline 40 & $\begin{array}{l}9.93 a \\
(1.36)\end{array}$ & $\begin{array}{l}0.40 \mathrm{a} \\
(0.03)\end{array}$ & $\begin{array}{l}197.47 b \\
(15.96)\end{array}$ & $\begin{array}{l}9.93 a \\
(1.36)\end{array}$ & $\begin{array}{l}0.77 \mathrm{c} \\
(0.17)\end{array}$ & $\begin{array}{c}97.49 a \\
(1.84)\end{array}$ & $\begin{array}{l}3.18 a \\
(0.34)\end{array}$ & $\begin{array}{l}18.65 b \\
(0.93)\end{array}$ & $\begin{array}{c}27.47 a \\
(5.87)\end{array}$ & $\begin{array}{l}38.27 \mathrm{~b} \\
(2.17)\end{array}$ & $\begin{array}{l}4.22 \mathrm{~b} \\
(0.76)\end{array}$ & $\begin{array}{l}11.73 \mathrm{c} \\
(1.70)\end{array}$ \\
\hline \multicolumn{13}{|l|}{ SS (rpm) } \\
\hline 100 & $\begin{array}{l}8.36 \mathrm{~b} \\
(0.81)\end{array}$ & $\begin{array}{l}0.41 a \\
(0.04)\end{array}$ & $\begin{array}{c}221.90 \mathrm{ab} \\
(11.70)\end{array}$ & $\begin{array}{l}8.36 b \\
(0.81)\end{array}$ & $\begin{array}{l}0.84 b \\
(0.16)\end{array}$ & $\begin{array}{c}94.78 a \\
(3.98)\end{array}$ & $\begin{array}{l}3.37 a \\
(0.39)\end{array}$ & $\begin{array}{c}19.29 a \\
(1.84)\end{array}$ & $\begin{array}{c}22.33 a \\
(9.07)\end{array}$ & $\begin{array}{c}42.30 a \\
(3.52)\end{array}$ & $\begin{array}{l}4.97 a \\
(1.03)\end{array}$ & $\begin{array}{c}14.22 \mathrm{a} \\
(1.51)\end{array}$ \\
\hline 150 & $\begin{array}{l}9.50 a \\
(1.09)\end{array}$ & $\begin{array}{l}0.40 a \\
(0.04)\end{array}$ & $\begin{array}{c}206.41 b \\
(19.77)\end{array}$ & $\begin{array}{l}9.5 a \\
(1.09)\end{array}$ & $\begin{array}{l}0.89 b \\
(0.16)\end{array}$ & $\begin{array}{c}94.16 a \\
(4.47)\end{array}$ & $\begin{array}{c}3.09 b \\
0.29\end{array}$ & $\begin{array}{l}19.17 a \\
(0.83)\end{array}$ & $\begin{array}{c}20.08 a \\
8.13\end{array}$ & $\begin{array}{l}43.28 a \\
(4.84)\end{array}$ & $\begin{array}{l}4.47 a \\
(0.74)\end{array}$ & $\begin{array}{c}13.62 \mathrm{a} \\
(2.13)\end{array}$ \\
\hline 200 & $\begin{array}{l}9.76 a \\
(1.47)\end{array}$ & $\begin{array}{l}0.40 a \\
(0.05)\end{array}$ & $\begin{array}{c}229.10 a \\
(34.27)\end{array}$ & $\begin{array}{l}9.76 a \\
(1.47)\end{array}$ & $\begin{array}{l}0.95 a \\
(0.10)\end{array}$ & $\begin{array}{l}94.85 a \\
(4.68)\end{array}$ & $\begin{array}{c}3.19 a b \\
(0.27)\end{array}$ & $\begin{array}{l}18.55 a \\
(0.91)\end{array}$ & $\begin{array}{l}20.92 a \\
(7.43)\end{array}$ & $\begin{array}{l}42.69 a \\
(4.86)\end{array}$ & $\begin{array}{l}4.45 a \\
(0.92)\end{array}$ & $\begin{array}{c}13.90 \mathrm{a} \\
(2.72)\end{array}$ \\
\hline \multicolumn{13}{|l|}{ Screw comp. } \\
\hline $2: 1$ & $\begin{array}{l}8.06 \mathrm{~b} \\
(0.56)\end{array}$ & $\begin{array}{l}0.46 a \\
(0.01)\end{array}$ & $\begin{array}{c}222.61 a \\
(29.52)\end{array}$ & $\begin{array}{l}8.06 b \\
(0.56)\end{array}$ & $\begin{array}{l}0.88 a \\
(0.15)\end{array}$ & $\begin{array}{c}95.91 a \\
(3.85)\end{array}$ & $\begin{array}{l}3.07 \mathrm{~b} \\
(0.24)\end{array}$ & $\begin{array}{l}18.68 a \\
(0.83)\end{array}$ & $\begin{array}{c}24.56 a \\
(6.26)\end{array}$ & $\begin{array}{l}42.30 a \\
(4.47)\end{array}$ & $\begin{array}{c}4.41 \\
(0.67)\end{array}$ & $\begin{array}{c}13.54 a \\
(1.98)\end{array}$ \\
\hline $3: 1$ & $\begin{array}{l}9.78 a \\
(1.16)\end{array}$ & $\begin{array}{l}0.37 \mathrm{~b} \\
(0.02)\end{array}$ & $\begin{array}{c}217.40 a \\
(23.07)\end{array}$ & $\begin{array}{l}9.78 a \\
(1.16)\end{array}$ & $\begin{array}{l}0.90 a \\
(0.14)\end{array}$ & $\begin{array}{c}93.94 a \\
(4.44)\end{array}$ & $\begin{array}{l}3.29 a \\
(0.35)\end{array}$ & $\begin{array}{c}19.17 a \\
(1.46)\end{array}$ & $\begin{array}{l}19.39 \mathrm{~b} \\
(8.48)\end{array}$ & $\begin{array}{c}42.98 a \\
(4.39)\end{array}$ & $\begin{array}{c}4.74 \\
(1.01)\end{array}$ & $\begin{array}{c}14.10 a \\
(2.23)\end{array}$ \\
\hline \multicolumn{13}{|l|}{ Die L/D } \\
\hline 5 & $\begin{array}{l}9.25 a \\
(1.13)\end{array}$ & $\begin{array}{l}0.40 a \\
(0.04)\end{array}$ & $\begin{array}{c}233.02 a \\
(21.60)\end{array}$ & $\begin{array}{l}9.25 a \\
(1.13)\end{array}$ & $\begin{array}{l}0.82 c \\
(0.22)\end{array}$ & $\begin{array}{c}91.35 \mathrm{~b} \\
(4.39)\end{array}$ & $\begin{array}{l}3.04 b \\
(0.27)\end{array}$ & $\begin{array}{c}19.53 a \\
(0.59)\end{array}$ & $\begin{array}{c}24.98 a \\
(7.81)\end{array}$ & $\begin{array}{c}45.76 a \\
(4.58)\end{array}$ & $\begin{array}{l}4.28 \mathrm{~b} \\
(0.47)\end{array}$ & $\begin{array}{c}14.38 \mathrm{a} \\
(1.41)\end{array}$ \\
\hline 9 & $\begin{array}{l}9.10 a \\
(1.49)\end{array}$ & $\begin{array}{l}0.41 a \\
(0.04)\end{array}$ & $\begin{array}{c}224.97 a b \\
(22.20)\end{array}$ & $\begin{array}{l}9.10 a \\
(1.49)\end{array}$ & $\begin{array}{l}0.90 \mathrm{~b} \\
(0.06)\end{array}$ & $\begin{array}{c}96.23 a \\
(1.89)\end{array}$ & $\begin{array}{c}3.24 a b \\
(0.39)\end{array}$ & $\begin{array}{c}18.92 \mathrm{ab} \\
(1.97)\end{array}$ & $\begin{array}{c}19.94 a b \\
(8.38)\end{array}$ & $\begin{array}{c}42.69 \mathrm{~b} \\
(3.41)\end{array}$ & $\begin{array}{c}4.57 a b \\
(0.89)\end{array}$ & $\begin{array}{c}13.56 a \\
(2.40)\end{array}$ \\
\hline 13 & $\begin{array}{l}9.26 a \\
(1.29)\end{array}$ & $\begin{array}{l}0.40 a \\
(0.04)\end{array}$ & $\begin{array}{c}199.42 \mathrm{~b} \\
(19.37)\end{array}$ & $\begin{array}{l}9.26 a \\
(1.29)\end{array}$ & $\begin{array}{l}0.96 a \\
(0.05)\end{array}$ & $\begin{array}{c}96.21 a \\
(4.30)\end{array}$ & $\begin{array}{l}3.37 a \\
(0.25)\end{array}$ & $\begin{array}{l}18.56 \mathrm{~b} \\
(0.71)\end{array}$ & $\begin{array}{l}18.42 \mathrm{a} \\
(7.07)\end{array}$ & $\begin{array}{c}39.82 \mathrm{c} \\
(2.96)\end{array}$ & $\begin{array}{l}5.03 a \\
(1.15)\end{array}$ & $\begin{array}{c}13.80 \mathrm{a} \\
(2.51)\end{array}$ \\
\hline \multicolumn{13}{|l|}{$\mathrm{T}\left({ }^{\circ} \mathrm{C}\right)$} \\
\hline 100 & $\begin{array}{l}9.03 a \\
(1.42)\end{array}$ & $\begin{array}{l}0.40 a \\
(0.05)\end{array}$ & $\begin{array}{c}221.36 a \\
(30.00)\end{array}$ & $\begin{array}{c}1160.89 a \\
(142.38)\end{array}$ & $\begin{array}{l}0.95 a \\
(0.06)\end{array}$ & $\begin{array}{c}94.47 a \\
(4.86)\end{array}$ & $\begin{array}{l}3.05 b \\
(0.26)\end{array}$ & $\begin{array}{c}18.95 a b \\
(1.09)\end{array}$ & $\begin{array}{l}23.42 \\
(7.08)\end{array}$ & $\begin{array}{c}42.91 a \\
(5.09)\end{array}$ & $\begin{array}{l}4.68 a \\
(0.74)\end{array}$ & $\begin{array}{c}14.24 a \\
(2.10)\end{array}$ \\
\hline 120 & $\begin{array}{l}9.64 a \\
(1.46)\end{array}$ & $\begin{array}{l}0.41 a \\
(0.04)\end{array}$ & $\begin{array}{c}219.75 a \\
(19.24)\end{array}$ & $\begin{array}{c}1185.14 a \\
(155.36)\end{array}$ & $\begin{array}{l}0.88 b \\
(0.18)\end{array}$ & $\begin{array}{c}94.59 a \\
(5.05)\end{array}$ & $\begin{array}{l}3.29 a \\
(0.26)\end{array}$ & $\begin{array}{c}18.59 \mathrm{~b} \\
(0.88)\end{array}$ & $\begin{array}{c}21.78 a \\
(7.57)\end{array}$ & $\begin{array}{c}42.83 a \\
(2.98)\end{array}$ & $\begin{array}{l}4.42 a \\
(0.86)\end{array}$ & $\begin{array}{c}13.48 \mathrm{a} \\
(2.40)\end{array}$ \\
\hline 140 & $\begin{array}{l}8.94 a \\
(0.85)\end{array}$ & $\begin{array}{l}0.40 a \\
(0.04)\end{array}$ & $\begin{array}{c}216.30 \mathrm{a} \\
(26.49)\end{array}$ & $\begin{array}{c}1096.63 b \\
(163.00)\end{array}$ & $\begin{array}{l}0.85 b \\
(0.16)\end{array}$ & $\begin{array}{c}94.73 a \\
(2.99)\end{array}$ & $\begin{array}{l}3.31 a \\
(0.41)\end{array}$ & $\begin{array}{c}19.47 a \\
(1.69)\end{array}$ & $\begin{array}{l}18.14 a \\
(9.13)\end{array}$ & $\begin{array}{c}42.52 a \\
(5.02)\end{array}$ & $\begin{array}{l}4.79 a \\
(1.13)\end{array}$ & $\begin{array}{c}14.02 \mathrm{a} \\
(1.98)\end{array}$ \\
\hline
\end{tabular}

* Means within a column (a given dependent variable) followed by similar letters for a given independent variable are not significantly different at $\mathrm{P}<0.05$, LSD. Values in parentheses are standard deviation. SBM is soybean meal; $\mathrm{MC}_{\mathrm{Raw}}$ is raw blend moisture content; $\mathrm{SS}$ is screw speed; Screw Comp. is screw compression ratio; die $\mathrm{L} / \mathrm{D}$ is length-to-diameter ratio of the die; $\mathrm{T}\left({ }^{\circ} \mathrm{C}\right)$ is temperature profile $\left(100\right.$ is $80-100-100^{\circ} \mathrm{C}, 120$ is $80-120-120,140$ is $\left.80-140-140^{\circ} \mathrm{C}\right) ; \mathrm{a}_{\mathrm{w} \text { Raw }}$ is water activity of raw blend; $\mathrm{L}_{\text {Raw }}$ is brightness/darkness of the raw blend; $\mathrm{a}_{\text {Raw }}$ is redness/greenness of the raw blend; $\mathrm{b}_{\text {Raw }}$ is yellowness/blueness of the raw blend; $\mathrm{P}$ is die pressure; MFR is mass flow rate; $\eta_{\text {app }}$ is dough apparent viscosity; SME is specific mechanical energy; $\mathrm{MC}_{\text {Ext }}$ is extrudate moisture content; $\mathrm{a}_{\mathrm{wExt}}$ is extrudate water activity; $\mathrm{BD}$ is extrudate bulk density; UD is extrudate unit density; ER is extrudate expansion ratio; PDI is extrudate pellet durability index; WAI is water adsorption index; WSI is water solubility index; $\mathrm{L}_{\mathrm{Ext}}$ is extrudate brightness/darkness; $\mathrm{a}_{\mathrm{Ext}}$ is extrudate redness/greenness; $b_{\mathrm{Ext}}$ is extrudate yellowness/blueness. 
Table 5. Treatment effects on the measured extrusion processing parameters*

\begin{tabular}{|c|c|c|c|c|c|c|c|c|c|c|c|c|c|c|c|c|c|c|}
\hline \multirow[b]{2}{*}{ Property } & \multicolumn{18}{|c|}{ Treatment } \\
\hline & 1 & 2 & 3 & 4 & 5 & 6 & 7 & 8 & 9 & 10 & 11 & 12 & 13 & 14 & 15 & 16 & 17 & 18 \\
\hline $\begin{array}{c}\mathrm{P} \text { at Die } \\
\text { (Mpa) }\end{array}$ & $\begin{array}{l}9.32 \mathrm{~b} \\
(2.06)\end{array}$ & $\begin{array}{l}5.03 \mathrm{ef} \\
(0.97)\end{array}$ & $\begin{array}{l}5.15 \mathrm{ef} \\
(1.71)\end{array}$ & $\begin{array}{l}11.05 a \\
(1.52)\end{array}$ & $\begin{array}{l}7.92 \mathrm{c} \\
(0.77)\end{array}$ & $\begin{array}{l}0.87 \mathrm{kl} \\
(0.28)\end{array}$ & $\begin{array}{l}9.12 \mathrm{~b} \\
(0.59)\end{array}$ & $\begin{array}{l}4.10 \mathrm{fg} \\
(0.08)\end{array}$ & $\begin{array}{l}9.90 \mathrm{~b} \\
(0.50)\end{array}$ & $\begin{array}{l}6.77 d \\
(1.63)\end{array}$ & $\begin{array}{l}2.55 i j \\
(0.38)\end{array}$ & $\begin{array}{l}1.53 \mathrm{jk} \\
(0.87)\end{array}$ & $\begin{array}{l}3.77 \mathrm{gh} \\
(0.45)\end{array}$ & $\begin{array}{l}4.11 \mathrm{fg} \\
(0.66)\end{array}$ & $\begin{array}{c}0.291 \\
(0.07)\end{array}$ & $\begin{array}{l}2.76 \mathrm{hi} \\
(0.76)\end{array}$ & $\begin{array}{l}5.56 \mathrm{e} \\
(1.50)\end{array}$ & $\begin{array}{l}7.91 \mathrm{c} \\
(1.60)\end{array}$ \\
\hline $\begin{array}{l}\text { Torque } \\
(\mathrm{Nm})\end{array}$ & $\begin{array}{c}31.67 \mathrm{bcd} \\
(2.27)\end{array}$ & $\begin{array}{c}26.81 \mathrm{c}-\mathrm{f} \\
(12.61)\end{array}$ & $\begin{array}{l}35.21 \mathrm{~b} \\
(9.46)\end{array}$ & $\begin{array}{l}44.05 \mathrm{~b} \\
(1.76)\end{array}$ & $\begin{array}{l}46.26 a \\
(4.62)\end{array}$ & $\begin{array}{l}8.26 \mathrm{~h} \\
(1.66)\end{array}$ & $\begin{array}{c}24.94 \mathrm{def} \\
(3.53)\end{array}$ & $\begin{array}{l}8.59 \mathrm{~h} \\
(0.31)\end{array}$ & $\begin{array}{l}22.93 \mathrm{ef} \\
(4.76)\end{array}$ & $\begin{array}{c}13.24 \mathrm{gh} \\
(4.39)\end{array}$ & $\begin{array}{c}20.20 \mathrm{fg} \\
(9.08)\end{array}$ & $\begin{array}{l}19.08 f g \\
(14.93)\end{array}$ & $\begin{array}{l}51.73 a \\
(16.60)\end{array}$ & $\begin{array}{c}29.66 \mathrm{~b}-\mathrm{e} \\
(10.04)\end{array}$ & $\begin{array}{l}8.38 \mathrm{~h} \\
(0.79)\end{array}$ & $\begin{array}{c}19.76 \mathrm{fg} \\
(8.90)\end{array}$ & $\begin{array}{l}23.99 \mathrm{def} \\
(5.08)\end{array}$ & $\begin{array}{c}33.14 \mathrm{bc} \\
(5.08)\end{array}$ \\
\hline $\begin{array}{c}\text { MFR } \\
\left(\mathrm{g} \mathrm{min}^{-1}\right)\end{array}$ & $\begin{array}{l}74.90 \mathrm{a} \\
(4.95)\end{array}$ & $\begin{array}{l}186.80 \mathrm{~b} \\
(50.95)\end{array}$ & $\begin{array}{c}225.60 \mathrm{a} \\
(7.88)\end{array}$ & $\begin{array}{c}172.60 \mathrm{bc} \\
(2.03)\end{array}$ & $\begin{array}{l}78.07 \mathrm{~g} \\
(0.64)\end{array}$ & $\begin{array}{l}72.07 \mathrm{~g} \\
(5.16)\end{array}$ & $\begin{array}{c}124.53 \mathrm{de} \\
(3.29)\end{array}$ & $\begin{array}{l}67.13 \mathrm{~g} \\
(2.19)\end{array}$ & $\begin{array}{c}88.00 f g \\
(9.10)\end{array}$ & $\begin{array}{c}\text { 105.73ef } \\
(4.01)\end{array}$ & $\begin{array}{l}85.67 \mathrm{fg} \\
(2.04)\end{array}$ & $\begin{array}{c}159.67 \mathrm{c} \\
(9.47)\end{array}$ & $\begin{array}{c}130.60 \mathrm{~d} \\
(4.86)\end{array}$ & $\begin{array}{c}161.20 \mathrm{c} \\
(0.53)\end{array}$ & $\begin{array}{l}75.53 \mathrm{~g} \\
(2.23)\end{array}$ & $\begin{array}{c}135.67 \mathrm{~d} \\
(3.83)\end{array}$ & $\begin{array}{l}76.53 \mathrm{~g} \\
(14.45)\end{array}$ & $\begin{array}{c}119.09 \mathrm{de} \\
(4.09)\end{array}$ \\
\hline$\eta_{\text {app }}$ & $2877.54 \mathrm{~b}$ & $1623.54 \mathrm{~cd}$ & $1599.64 \mathrm{~cd}$ & $1697.67 \mathrm{~cd}$ & $3565.88 a$ & 424.44h & $1510.01 \mathrm{cde}$ & 762.06fgh & $1388.15 \mathrm{de}$ & 601.36gh & $1835.30 \mathrm{c}$ & $866.54 \mathrm{fg}$ & $2658.80 \mathrm{~b}$ & 1143.18ef & $645.54 \mathrm{gh}$ & $761.64 \mathrm{fgh}$ & $1848.94 \mathrm{c}$ & $1703.35 \mathrm{~cd}$ \\
\hline (Pa.s) & (205.99) & (763.30) & (429.84) & $(67.92)$ & (356.36) & (85.74) & $(213.96)$ & (88.28) & (288.26) & (199.37) & (824.80) & (678.28) & (853.39) & (386.99) & $(61.07)$ & (343.18) & (391.46) & (261.15) \\
\hline $\begin{array}{l}\text { SME } \\
\left(\mathrm{J} \mathrm{g}^{-1}\right)\end{array}$ & $\begin{array}{c}755.96 \mathrm{bcd} \\
(10.71)\end{array}$ & $\begin{array}{l}582.70 \mathrm{c}-f \\
(36.24)\end{array}$ & $\begin{array}{c}784.55 \mathrm{bcd} \\
(66.08)\end{array}$ & $\begin{array}{c}830.24 \mathrm{bc} \\
(18.24)\end{array}$ & $\begin{array}{c}1250.33 a \\
(88.99)\end{array}$ & $\begin{array}{c}411.01 \text { def } \\
\text { (75.12) }\end{array}$ & $\begin{array}{c}\text { 615.51c-f } \\
(59.62)\end{array}$ & $\begin{array}{c}297.70 f \\
(3.35)\end{array}$ & $\begin{array}{c}473.70 c-f \\
(46.00)\end{array}$ & $\begin{array}{c}350.50 \mathrm{ef} \\
(14.80)\end{array}$ & $\begin{array}{l}\text { 408.10def } \\
\text { (192.87) }\end{array}$ & $\begin{array}{c}\text { f1055.00at } \\
(809.03)\end{array}$ & $\begin{array}{c}1449.40 \mathrm{a} \\
(469.37)\end{array}$ & $\begin{array}{r}831.80 \mathrm{bc} \\
(252.57)\end{array}$ & $\begin{array}{c}353.20 \mathrm{ef} \\
(42.07)\end{array}$ & $\begin{array}{c}756.00 \mathrm{bcd} \\
(138.74)\end{array}$ & $\begin{array}{c}d 606.90 c-f \\
(96.15)\end{array}$ & $\begin{array}{c}732.50 \mathrm{~b}-\mathrm{e} \\
(175.40)\end{array}$ \\
\hline $\begin{array}{l}\mathrm{T} 1 \\
\left({ }^{\circ} \mathrm{C}\right)\end{array}$ & $\begin{array}{l}80.75 \\
(1.39)\end{array}$ & $\begin{array}{l}78.63 \\
(3.34)\end{array}$ & $\begin{array}{l}81.25 \\
(2.55)\end{array}$ & $\begin{array}{l}79.38 \\
(0.52)\end{array}$ & $\begin{array}{l}80.75 \\
(1.83)\end{array}$ & $\begin{array}{l}78.80 \\
(1.98)\end{array}$ & $\begin{array}{l}79.25 \\
(0.71)\end{array}$ & $\begin{array}{l}79.75 \\
(0.46)\end{array}$ & $\begin{array}{l}80.25 \\
(0.71)\end{array}$ & $\begin{array}{l}80.00 \\
(0.53)\end{array}$ & $\begin{array}{l}80.00 \\
(0.76)\end{array}$ & $\begin{array}{l}82.13 \\
(1.96)\end{array}$ & $\begin{array}{l}82.13 \\
(2.10)\end{array}$ & $\begin{array}{l}81.00 \\
(2.51)\end{array}$ & $\begin{array}{l}80.63 \\
(0.74)\end{array}$ & $\begin{array}{l}79.63 \\
(0.52)\end{array}$ & $\begin{array}{l}79.75 \\
(0.71)\end{array}$ & $\begin{array}{l}79.63 \\
(0.52)\end{array}$ \\
\hline $\begin{array}{c}\mathrm{T} 2 \\
\left({ }^{\circ} \mathrm{C}\right)\end{array}$ & $\begin{array}{l}101.38 \\
(0.91)\end{array}$ & $\begin{array}{l}118.88 \\
(0.35)\end{array}$ & $\begin{array}{l}139.13 \\
(0.83)\end{array}$ & $\begin{array}{l}102.75 \\
(4.50)\end{array}$ & $\begin{array}{l}121.00 \\
(0.53)\end{array}$ & $\begin{array}{l}140.25 \\
(0.46)\end{array}$ & $\begin{array}{l}99.75 \\
(0.46)\end{array}$ & $\begin{array}{l}100.00 \\
(0.00)\end{array}$ & $\begin{array}{l}122.50 \\
(1.19)\end{array}$ & $\begin{array}{c}119.38 \\
(0.92)\end{array}$ & $\begin{array}{l}140.00 \\
(1.07)\end{array}$ & $\begin{array}{l}133.00 \\
(8.64)\end{array}$ & $\begin{array}{l}100.88 \\
(0.64)\end{array}$ & $\begin{array}{l}96.88 \\
(3.87)\end{array}$ & $\begin{array}{l}122.38 \\
(3.85)\end{array}$ & $\begin{array}{c}119.63 \\
(0.74)\end{array}$ & $\begin{array}{c}139.88 \\
(0.35)\end{array}$ & $\begin{array}{l}138.00 \\
(1.07)\end{array}$ \\
\hline T3 & $\begin{array}{l}103.00 \\
103.1)\end{array}$ & 119.00 & & & & & & & & & & & & & 121.88 & & 139.88 & 140.63 \\
\hline$\left({ }^{\circ} \mathrm{C}\right)$ & $(2.20)$ & $(0.76)$ & $(0.74)$ & $(3.10)$ & (1.73) & (1.41) & $(0.53)$ & $(0.00)$ & $(1.31)$ & $(0.46)$ & $(0.00)$ & $(4.41)$ & $(0.35)$ & $(2.05)$ & (2.53) & $(0.00)$ & $(0.35)$ & $(1.85)$ \\
\hline
\end{tabular}

* Means followed by similar letters for a given dependent variable (row) are not significantly different at $\mathrm{P}<0.05$, LSD. Values in parentheses are standard deviation. $\mathrm{P}$ is die pressure, MFR is mass flow rate, $\eta_{\text {app }}$ is apparent viscosity, SME is specific mechanical energy, T1 is extruder feed zone temperature, T2 is extruder metering zone temperature, $\mathrm{T} 3$ is extruder die zone temperature.

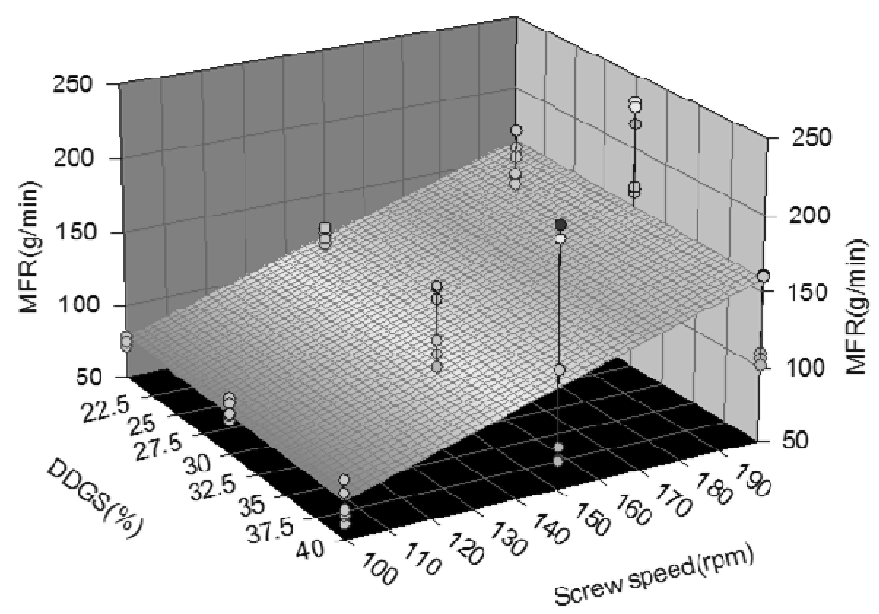

Figure 1. Treatment combination effects on mass flow rate

$\operatorname{MFR}(\mathrm{g} / \mathrm{min})=-1.19-0.19 * \mathrm{DDGS}(\mathrm{g} / \mathrm{kg})+0.84 * \mathrm{SS}(\mathrm{rpm}) ;\left(\mathrm{R}^{2}=0.54, \mathrm{~F}=29.58\right)$.

\subsubsection{Apparent Viscosity}

All independent variables had significant effects on the apparent viscosity, as shown by the main and treatment effects (Tables 4 and 5). Standard deviations for the apparent viscosity were somewhat high; values for apparent viscosity ranged between 424.44 (Run 6) and 3565.88 (Run 5) Pa.s. For the main effects (Table 4), with increasing DDGS content, temperature, screw speed, and screw compression ratio, decreases in apparent viscosity were observed. Raising the DDGS level from 20 to $40 \%$ yielded a decrease in apparent viscosity of $43.3 \%$. Similar results were observed by Kannadhason et al. (2009b). Compared to soybean meal, DDGS has higher fiber content and less protein. Increasing the DDGS content in the blends, while reducing the amount of SBM, changed the chemical composition and the potential functionality of the ingredients in the dough, thus affecting the apparent viscosity (Chevanan et al., 2010). A decrease in apparent viscosity by $22.3 \%$ was observed as the die zone temperature was raised from 100 to $140^{\circ} \mathrm{C}$; this result is supported by findings reported by Kannadhason et al. (2009b) and Chevanan et al. (2010). Likewise, previous studies reported that increasing the temperature resulted in a decrease in viscosity (Launay \& Lisch, 1983; Senouci \& Smith, 1988). The reduced viscosity can be related to starch gelatinization, protein denaturation, chemical and structural transformations, respectively. Changes due to higher moisture content were curvilinear and showed a significant difference only when increasing MC from 30 to $40 \%$, specifically a reduction of $51.2 \%$. Similar findings were made by Chevanan et al. (2007a, 2010), who concluded that this behavior was caused by competing interactions between moisture content and other independent variables. Increasing the screw speed from 100 to 150 and $200 \mathrm{rpm}$ had significant effects on all recorded values for the apparent viscosity, and yielded an overall decrease by $42.3 \%$. These recordings are similar to observations made by other researchers (Kannadhason et al., 2009b), as the dough was pseudoplastic. On the other hand, an increase in viscosity of $36.9 \%$ was observed when increasing the die L/D ratio from 5 to 13 . 
Chevanan et al. (2007a) made similar observations as well. With changes in die L/D ratio, only the die diameter changed, whereas the length stayed the same. With a smaller die diameter, pressure and shear increased, which led to an increase in viscosity. Increasing the screw compression led to a decrease in apparent viscosity by $33.3 \%$, and was pseudoplastic behavior.

\subsubsection{Specific Mechanical Energy}

Specific mechanical energy consumption quantifies the net energy that is required to convey the material through the extruder per unit rate of mass flow. For the main effects, no clear pattern of changes could be observed with varying levels of the independent variables, which again was related to the high standard deviations. The highest SME was detected at $1449.40 \mathrm{~J} / \mathrm{g}$ (Run 13) and the lowest at $297.70 \mathrm{~J} / \mathrm{g}$ (Run 8; Table 5). With increasing DDGS level, SME showed a significant decrease (Table 4). With increasing SBM, however, the SME increased as well. Increasing the screw speed and compression ratio of the screw yielded an increase and decrease in SME by $25.4 \%$ and $14 \%$, respectively. As temperature profile and L/D ratio increased, SME exhibited curvilinear behavior.

\subsubsection{Temperature}

Temperature settings were adjusted in the beginning of each extrusion run to the desired value. However, throughout processing, temperatures within the different zones increased due to friction (Table 5) and were adjusted by using external air when temperature increased more than $5^{\circ} \mathrm{C}$. These temperature effects were expected, due to frictional heating and shear forces in the barrel during extrusion processing. This was due to the design of the extruder (i.e. the conveying mechanism of the flighted screw, the viscous properties of the raw material, and the grooved walls that reduce slip and cause friction) (Harper, 1981).

\subsection{Extrudate Physical Properties}

\subsubsection{Moisture Content}

The moisture content of the raw blends had one of the most important impacts on almost all extrudate physical properties and their cohesiveness (Table 4). Previous studies have shown that extrudate MC increased with higher DDGS levels (Ayadi et al., 2011b; Kannadhason et al., 2010) as well as the MC of the raw blends (Kannadhason et al., 2009b; Rosentrater, 2009b). In this study, extrudate MC decreased significantly (by 8.8\%) when DDGS content of the blends increased from 20 to $30 \%$, whereas MC did not show significant effects when increasing DDGS level from 30 to $40 \%$. Increases with higher initial MC were as expected due to the higher water content of the raw blends. The difference between initial and final $\mathrm{MC}$ can be caused by greater flashing of moisture during exiting the die. The highest value for extrudate MC was $12.01 \%$ (Run 16) and the lowest was $7.24 \%$ (Run 1) (Table 6). Regarding the other independent variables, some differences occurred for the extrudate MC, in terms of main effect or treatment combination effects (Table 6). Also, using the 3:1 screw (with a higher compression ratio) yielded higher extrudate MC, by $21.3 \%$.

Table 6. Treatment effects on extrudate physical properties*

\begin{tabular}{|c|c|c|c|c|c|c|c|c|c|c|c|c|c|c|c|c|c|c|}
\hline \multirow[b]{2}{*}{ Property } & \multicolumn{18}{|c|}{ Treatment } \\
\hline & 1 & 2 & 3 & 4 & 5 & 6 & 7 & 8 & 9 & 10 & 11 & 12 & 13 & 14 & 15 & 16 & 17 & 18 \\
\hline MC & $7.24 \mathrm{k}$ & $8.10 \mathrm{i}$ & $8.62 \mathrm{gh}$ & $7.67 \mathrm{j}$ & $7.88 \mathrm{ij}$ & $8.84 \mathrm{fg}$ & $11.43 \mathrm{~b}$ & $9.27 \mathrm{e}$ & $10.12 \mathrm{~d}$ & $10.28 \mathrm{~cd}$ & $8.22 \mathrm{hi}$ & $10.54 \mathrm{c}$ & $9.19 \mathrm{ef}$ & $9.41 \mathrm{e}$ & $9.43 \mathrm{e}$ & 12.01a & $8.11 \mathrm{i}$ & $9.33 \mathrm{e}$ \\
\hline$\left(\mathrm{g} \mathrm{kg}^{-1}\right)$ & $(0.09)$ & $(0.05)$ & $(0.10)$ & $(0.06)$ & $(0.11)$ & $(0.04)$ & $(0.04)$ & $(0.32)$ & $(0.21)$ & $(0.32)$ & $(0.05)$ & $(0.23)$ & $(0.29)$ & $(0.58)$ & (0.31) & $(0.45)$ & $(0.12)$ & $(0.13)$ \\
\hline$a_{w}$ & $0.47 a$ & $0.45 c$ & $0.44 \mathrm{c}$ & $0.47 a$ & $0.46 \mathrm{~b}$ & $0.45 \mathrm{c}$ & $0.38 f$ & $0.37 \mathrm{gh}$ & $0.38 \mathrm{fg}$ & $0.37 \mathrm{fgh}$ & $0.39 e$ & $0.37 \mathrm{gh}$ & $0.37 \mathrm{gh}$ & $0.35 \mathrm{i}$ & $0.38 \mathrm{fg}$ & $0.41 \mathrm{~d}$ & $0.37 \mathrm{fg}$ & $0.36 \mathrm{~h}$ \\
\hline$(-)$ & $(0.00)$ & $(0.00)$ & $(0.01)$ & $(0.00)$ & $(0.00)$ & $(0.00)$ & $(0.01)$ & $(0.00)$ & $(0.00)$ & $(0.00)$ & $(0.01)$ & $(0.00)$ & $(0.00)$ & $(0.00)$ & $(0.00)$ & $(0.00)$ & $(0.01)$ & $(0.00)$ \\
\hline $\begin{array}{c}\mathrm{BD} \\
\left(\mathrm{kg} \mathrm{m}^{-3}\right)\end{array}$ & $\begin{array}{c}241.22 \mathrm{~d} \\
(2.08)\end{array}$ & $\begin{array}{c}230.81 \mathrm{e} \\
(2.10)\end{array}$ & $\begin{array}{c}177.101 \\
(4.78)\end{array}$ & $\begin{array}{c}267.39 a \\
(1.42)\end{array}$ & $\begin{array}{c}215.98 \mathrm{~g} \\
(2.19)\end{array}$ & $\begin{array}{c}203.16 \mathrm{j} \\
(1.41)\end{array}$ & $\begin{array}{c}175.04 \mathrm{I} \\
(2.56)\end{array}$ & $\begin{array}{c}209.17 \mathrm{hi} \\
(3.11)\end{array}$ & $\begin{array}{c}196.62 \mathrm{k} \\
(3.36)\end{array}$ & $\begin{array}{c}254.73 c \\
(0.66)\end{array}$ & $\begin{array}{c}228.93 \mathrm{ef} \\
(2.48)\end{array}$ & $\begin{array}{c}259.34 \mathrm{~b} \\
(1.24)\end{array}$ & $\begin{array}{c}228.85 \text { ef } \\
(0.74)\end{array}$ & $\begin{array}{c}206.49 \mathrm{ij} \\
(1.40)\end{array}$ & $\begin{array}{c}210.82 \mathrm{~h} \\
(2.76)\end{array}$ & $\begin{array}{c}209.55 \mathrm{hi} \\
(0.28)\end{array}$ & $\begin{array}{c}225.29 f \\
(0.40)\end{array}$ & $\begin{array}{c}204.00 \mathrm{j} \\
(1.89)\end{array}$ \\
\hline$\underset{\left(\mathrm{kg} \mathrm{m}^{-3}\right)}{U D}$ & $\begin{array}{c}1012.01 \mathrm{ij} \\
(58.16)\end{array}$ & $\begin{array}{c}1132.85 \mathrm{eh} \\
(51.00)\end{array}$ & $\begin{array}{c}1254.34 \mathrm{bcd} \\
(49.20)\end{array}$ & $\begin{array}{c}1109.81 \mathrm{ei} \\
(50.00)\end{array}$ & $\begin{array}{c}1329.95 a b \\
(200.92)\end{array}$ & $\begin{array}{c}1170.72 \mathrm{def} \\
(167.36)\end{array}$ & $\begin{array}{c}1389.22 a \\
(65.73)\end{array}$ & $\begin{array}{c}1257.15 \mathrm{bcd} \\
(62.34)\end{array}$ & $\begin{array}{l}1182.74 \text { cde } \\
\text { (55.12) }\end{array}$ & $\begin{array}{c}1023.55 i \\
(27.95)\end{array}$ & $\begin{array}{c}925.42 \mathrm{j} \\
(148.21)\end{array}$ & $\begin{array}{c}1081.64 f i \\
(43.24)\end{array}$ & $\begin{array}{c}1045.62 \mathrm{i} \\
(52.83)\end{array}$ & $\begin{array}{c}1153.98 \mathrm{efg} \\
(75.80)\end{array}$ & $\begin{array}{c}1164.84 \mathrm{dg} \\
(198.15)\end{array}$ & $\begin{array}{c}1279.89 \mathrm{bc} \\
(80.01)\end{array}$ & 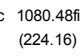 & $\begin{array}{c}1068.86 \mathrm{ghi} \\
(46.30)\end{array}$ \\
\hline $\begin{array}{c}\text { ER } \\
(-)\end{array}$ & $\begin{array}{l}0.94 \mathrm{de} \\
(0.04)\end{array}$ & $\begin{array}{l}0.91 \mathrm{e} \\
(0.02)\end{array}$ & $\begin{array}{l}0.94 \mathrm{de} \\
(0.02)\end{array}$ & $\begin{array}{l}0.99 \mathrm{bc} \\
(0.03)\end{array}$ & $\begin{array}{l}0.94 \mathrm{de} \\
(0.02)\end{array}$ & $\begin{array}{l}0.56 \mathrm{~g} \\
(0.03)\end{array}$ & $\begin{array}{l}0.90 \mathrm{e} \\
(0.01)\end{array}$ & $\begin{array}{l}0.85 f \\
(0.02)\end{array}$ & $\begin{array}{l}1.02 \mathrm{ab} \\
(0.03)\end{array}$ & $\begin{array}{l}1.05 a \\
(0.02)\end{array}$ & $\begin{array}{l}0.82 f \\
(0.02)\end{array}$ & $\begin{array}{l}0.84 f \\
(0.15)\end{array}$ & $\begin{array}{l}0.99 b c \\
(0.01)\end{array}$ & $\begin{array}{l}0.99 \mathrm{bc} \\
(0.03)\end{array}$ & $\begin{array}{l}0.53 \mathrm{~g} \\
(0.07)\end{array}$ & $\begin{array}{l}0.85 f \\
(0.03)\end{array}$ & $\begin{array}{l}0.97 \mathrm{~cd} \\
(0.06)\end{array}$ & $\begin{array}{l}0.94 \mathrm{de} \\
(0.05)\end{array}$ \\
\hline $\begin{array}{l}\text { PDI } \\
(\%)\end{array}$ & $\begin{array}{c}\text { 87.79gh } \\
(0.20)\end{array}$ & $\begin{array}{c}96.07 a-e \\
(0.11)\end{array}$ & $\begin{array}{c}98.99 a \\
(0.17)\end{array}$ & $\begin{array}{c}97.61 \mathrm{abc} \\
(0.08)\end{array}$ & $\begin{array}{c}97.95 \mathrm{abc} \\
(0.01)\end{array}$ & $\begin{array}{c}97.07 a-d \\
(0.05)\end{array}$ & $\begin{array}{c}96.35 a-e \\
(4.60)\end{array}$ & $\begin{array}{c}98.44 a b \\
(0.06)\end{array}$ & $\begin{array}{l}\text { 92.93ef } \\
(8.71)\end{array}$ & $\begin{array}{l}86.49 \mathrm{~h} \\
(0.30)\end{array}$ & $\begin{array}{c}94.38 \mathrm{~b}-\mathrm{f} \\
(2.29)\end{array}$ & $\begin{array}{l}91.01 \mathrm{fg} \\
(0.53)\end{array}$ & $\begin{array}{c}88.70 \mathrm{gh} \\
(0.76)\end{array}$ & $\begin{array}{c}97.94 \mathrm{abc} \\
(0.30)\end{array}$ & $\begin{array}{c}97.04 a-e \\
(0.06)\end{array}$ & $\begin{array}{c}97.07 a-d \\
(0.23)\end{array}$ & $\begin{array}{c}93.11 \mathrm{def} \\
(2.97)\end{array}$ & $\begin{array}{c}93.83 \mathrm{c}-\mathrm{f} \\
(0.27)\end{array}$ \\
\hline $\begin{array}{c}\text { WAI } \\
(-)\end{array}$ & $\begin{array}{l}3.00 \mathrm{i} \\
(0.01)\end{array}$ & $\begin{array}{c}\text { 3.04ghi } \\
(0.04)\end{array}$ & $\begin{array}{l}3.03 \mathrm{hi} \\
(0.02)\end{array}$ & $\begin{array}{l}\text { 3.05gh } \\
(0.02)\end{array}$ & $\begin{array}{l}3.55 d \\
(0.01)\end{array}$ & $\begin{array}{l}2.77 \mathrm{k} \\
(0.02)\end{array}$ & $\begin{array}{l}3.55 d \\
(0.01)\end{array}$ & $\begin{array}{l}2.81 \mathrm{k} \\
(0.02)\end{array}$ & $\begin{array}{l}3.33 f \\
(0.00)\end{array}$ & $\begin{array}{l}2.90 \mathrm{j} \\
(0.01)\end{array}$ & $\begin{array}{l}3.90 \mathrm{a} \\
(0.02)\end{array}$ & $\begin{array}{l}3.48 \mathrm{e} \\
(0.06)\end{array}$ & $\begin{array}{l}2.81 \mathrm{k} \\
(0.00)\end{array}$ & $\begin{array}{l}3.08 \mathrm{~g} \\
(0.06)\end{array}$ & $\begin{array}{l}3.30 f \\
(0.01)\end{array}$ & $\begin{array}{l}3.60 \mathrm{c} \\
(0.01)\end{array}$ & $\begin{array}{l}3.67 \mathrm{~b} \\
(0.01)\end{array}$ & $\begin{array}{c}\text { 3.04ghi } \\
(0.01)\end{array}$ \\
\hline $\begin{array}{l}\text { WSI } \\
(\%)\end{array}$ & $\begin{array}{l}20.10 \mathrm{~b} \\
(0.15)\end{array}$ & $\begin{array}{c}17.72 \mathrm{gh} \\
(0.40)\end{array}$ & $\begin{array}{c}18.56 \mathrm{efg} \\
(0.22)\end{array}$ & $\begin{array}{c}17.98 \mathrm{fgh} \\
(0.05)\end{array}$ & $\begin{array}{c}\text { 18.64def } \\
(0.08)\end{array}$ & $\begin{array}{c}19.05 \mathrm{cde} \\
(0.50)\end{array}$ & $\begin{array}{c}19.51 \mathrm{bc} \\
(0.18)\end{array}$ & $\begin{array}{c}\text { 17.73gh } \\
(0.55)\end{array}$ & $\begin{array}{c}19.06 \mathrm{cde} \\
(0.03)\end{array}$ & $\begin{array}{c}\text { 19.10cde } \\
(0.71)\end{array}$ & $\begin{array}{c}22.72 a \\
(0.09)\end{array}$ & $\begin{array}{c}19.58 \mathrm{bc} \\
(0.11)\end{array}$ & $\begin{array}{c}20.24 \mathrm{~b} \\
(0.17)\end{array}$ & $\begin{array}{c}\text { 18.16fgh } \\
(0.45)\end{array}$ & $\begin{array}{c}\text { 19.10cde } \\
(0.22)\end{array}$ & $\begin{array}{c}17.93 \text { fgh } \\
(1.72)\end{array}$ & $\begin{array}{c}17.43 \mathrm{~h} \\
(0.16)\end{array}$ & $\begin{array}{c}19.45 \mathrm{bcd} \\
(0.45)\end{array}$ \\
\hline $\begin{array}{l}\text { WS } \\
\text { (min) }\end{array}$ & $\begin{array}{c}>30.00 a \\
(0.00)\end{array}$ & $\begin{array}{l}19.67 \mathrm{c} \\
(4.73)\end{array}$ & $\begin{array}{c}>30.00 \mathrm{a} \\
(0.00)\end{array}$ & $\begin{array}{c}\text { 15.33de } \\
(0.58)\end{array}$ & $\begin{array}{c}22.33 \mathrm{~b} \\
(2.52)\end{array}$ & $\begin{array}{c}>30.00 a \\
(0.00)\end{array}$ & $\begin{array}{c}\text { 14.83de } \\
(2.25)\end{array}$ & $\begin{array}{c}>30.00 a \\
(0.00)\end{array}$ & $\begin{array}{l}9.17 \mathrm{f} \\
(1.04)\end{array}$ & $\begin{array}{l}19.50 \mathrm{c} \\
(0.87)\end{array}$ & $\begin{array}{l}7.83 f \\
(1.53)\end{array}$ & $\begin{array}{l}10.37 f \\
(1.10)\end{array}$ & $\begin{array}{c}>30.00 \mathrm{a} \\
(0.00)\end{array}$ & $\begin{array}{c}20.33 \mathrm{~b} \\
(1.53)\end{array}$ & $\begin{array}{c}>30.00 a \\
(0.00)\end{array}$ & $\begin{array}{c}>30.00 \mathrm{a} \\
(0.00)\end{array}$ & $\begin{array}{l}13.83 \mathrm{e} \\
(1.26)\end{array}$ & $\begin{array}{l}16.83 \mathrm{~d} \\
(1.26)\end{array}$ \\
\hline $\begin{array}{l}\mathrm{L} \\
(-)\end{array}$ & $\begin{array}{c}48.96 \mathrm{a} \\
(0.35)\end{array}$ & $\begin{array}{r}43.94 \mathrm{~b} \\
(1.19)\end{array}$ & $\begin{array}{c}35.55 \mathrm{~g} \\
(0.92)\end{array}$ & $\begin{array}{c}43.53 \mathrm{~b} \\
(0.19)\end{array}$ & $\begin{array}{c}42.61 \mathrm{bc} \\
(1.42)\end{array}$ & $\begin{array}{c}39.20 \mathrm{de} \\
(3.18)\end{array}$ & $\begin{array}{c}36.53 \mathrm{fg} \\
(0.37)\end{array}$ & $\begin{array}{c}38.42 \mathrm{ef} \\
(0.92)\end{array}$ & $\begin{array}{c}42.44 \mathrm{bc} \\
(0.54)\end{array}$ & $\begin{array}{c}48.10 a \\
(0.55)\end{array}$ & $\begin{array}{c}42.44 \mathrm{bc} \\
(1.94)\end{array}$ & $\begin{array}{c}48.64 a \\
(1.19)\end{array}$ & $\begin{array}{c}49.40 \mathrm{a} \\
(0.23)\end{array}$ & $\begin{array}{c}40.66 \mathrm{~cd} \\
(1.04)\end{array}$ & $\begin{array}{c}40.25 \mathrm{de} \\
(0.10)\end{array}$ & $\begin{array}{c}\text { 39.64de } \\
(1.56)\end{array}$ & $\begin{array}{c}41.11 \mathrm{~cd} \\
(1.11)\end{array}$ & $\begin{array}{c}48.18 \mathrm{a} \\
(0.37)\end{array}$ \\
\hline $\begin{array}{l}a \\
(-)\end{array}$ & $\begin{array}{l}3.96 \mathrm{e} \\
(0.16)\end{array}$ & $\begin{array}{c}\text { 4.54cde } \\
(0.80)\end{array}$ & $\begin{array}{l}3.87 \mathrm{ef} \\
(0.19)\end{array}$ & $\begin{array}{l}5.39 \mathrm{~b} \\
(0.07)\end{array}$ & $\begin{array}{l}4.15 \mathrm{e} \\
(0.21)\end{array}$ & $\begin{array}{c}4.53 \mathrm{cde} \\
(0.89)\end{array}$ & $\begin{array}{l}4.04 \mathrm{e} \\
(0.11)\end{array}$ & $\begin{array}{c}5.05 \mathrm{bcd} \\
(0.25)\end{array}$ & $\begin{array}{l}5.61 \mathrm{~b} \\
(0.05)\end{array}$ & $\begin{array}{l}4.40 \mathrm{de} \\
(0.39)\end{array}$ & $\begin{array}{l}5.15 b c \\
(0.39)\end{array}$ & $\begin{array}{l}4.20 \mathrm{e} \\
(0.16)\end{array}$ & $\begin{array}{l}4.01 e \\
(0.48)\end{array}$ & $\begin{array}{l}5.60 \mathrm{~b} \\
(0.20)\end{array}$ & $\begin{array}{c}4.58 \mathrm{cde} \\
(0.40)\end{array}$ & $\begin{array}{l}3.21 \mathrm{f} \\
(0.88)\end{array}$ & $\begin{array}{l}6.92 a \\
(0.24)\end{array}$ & $\begin{array}{l}4.06 \mathrm{e} \\
(0.38)\end{array}$ \\
\hline $\begin{array}{l}\mathrm{b} \\
(-)\end{array}$ & $\begin{array}{c}14.84 \mathrm{bcd} \\
(0.47)\end{array}$ & $\begin{array}{c}\text { 13.63de } \\
(1.85)\end{array}$ & $\begin{array}{c}11.26 \mathrm{fg} \\
(0.44)\end{array}$ & $\begin{array}{c}16.47 a b \\
(0.11) \\
\end{array}$ & $\begin{array}{c}12.59 \text { ef } \\
(0.45)\end{array}$ & $\begin{array}{c}12.46 \mathrm{ef} \\
(1.72)\end{array}$ & $\begin{array}{c}10.58 \mathrm{gh} \\
(0.22)\end{array}$ & $\begin{array}{r}12.98 \mathrm{e} \\
(0.57) \\
\end{array}$ & $\begin{array}{c}16.21 \mathrm{ab} \\
(0.06)\end{array}$ & $\begin{array}{c}15.33 \mathrm{abc} \\
(1.03)\end{array}$ & $\begin{array}{c}14.85 \mathrm{bcd} \\
(1.09)\end{array}$ & $\begin{array}{c}15.15 \mathrm{a}-\mathrm{d} \\
(0.08)\end{array}$ & $\begin{array}{c}14.99 \mathrm{a}-\mathrm{d} \\
(1.51)\end{array}$ & $\begin{array}{c}15.60 \mathrm{ab} \\
(0.64)\end{array}$ & $\begin{array}{c}13.52 \mathrm{de} \\
(0.75)\end{array}$ & $\begin{array}{l}9.60 \mathrm{~h} \\
(1.88)\end{array}$ & $\begin{array}{c}16.56 a \\
(0.61)\end{array}$ & $\begin{array}{c}13.83 \mathrm{cde} \\
(1.02)\end{array}$ \\
\hline
\end{tabular}


* Means followed by similar letters for a given dependent variable (row) are not significantly different at $\mathrm{P}<0.05$, LSD. Values in parentheses are standard deviation. MC is moisture content, $\mathrm{a}_{\mathrm{w}}$ is water activity, BD is bulk density, UD is unit density, ER is expansion ratio, PDI is pellet durability index, WAI is water absorption index, WSI is water solubility index, WS is water stability; L is brightness/darkness of extrudate; a is redness/greenness of extrudate; $b$ is yellowness/ blueness of extrudate.

\subsubsection{Water Activity}

Water activity measures the free water that is unbound in a material and can be available for microorganisms such as bacteria, molds, and yeast. A critical aw exists for every microorganism, below which growth is inhibited. In contrast to bacteria, yeasts and molds can reproduce at lower $\mathrm{a}_{\mathrm{w}}$. Generally, $\mathrm{a}_{\mathrm{w}}$ below 0.60 sufficiently restricts microbial growth and reduces the risk of deterioration (Chirife \& Del Pilar Buera, 1994; Lowe \& Kershaw, 1995).

Regarding main effects, standard deviations for the raw blends were relatively low and significant differences occurred only due to higher moisture content levels. Water activity increased by $25.4 \%$ when raising the moisture content from 20 to $40 \%$. Water activity of the extrudate did not exhibit any significant differences due to processing conditions or ingredient composition, except for different screw configuration. Significant differences for the extrudates occurred only due to screw configuration: aw decreased by $19.6 \%$ from 0.46 to 0.37 when increasing the compression ratio of the screw (Table 4). Water activity for the raw material ranged between 0.62 and 0.80 (Table 3 ) and between 0.35 and 0.47 for the final product (Table 6).

\subsubsection{Bulk Density}

Bulk density is an important parameter for the design of storage vessels. It determines the required storage space for the processing plant or shipping (Guy, 2001). High values for BD imply a higher capacity of extrudates which can be stored in a container. Bulk density varied between 175.04 and $267.39 \mathrm{~kg} / \mathrm{m}^{3}$ for the extrudates (Table 6). Concerning the main effects (Table 4), some significant differences were detected due to varying moisture content, screw speed, and die L/D ratio. A significant decrease for $\mathrm{BD}$, by $13.5 \%$, was detected when increasing $\mathrm{MC}$ from 30 to $40 \%$. Raising the screw speed from 100 to $150 \mathrm{rpm}$ resulted first in a decrease in BD by $7.0 \%$, and then in an increase by $11.0 \%$ when increasing the screw speed from 150 to $200 \mathrm{rpm}$. Bulk density decreased by $14.4 \%$ with a higher die L/D ratio; a significant difference was observed between the highest and lowest die L/D ratio (Table 6).

\subsubsection{Unit Density}

Unit density ranged from 925.42 to $1389.22 \mathrm{~kg} / \mathrm{m}^{3}$ (Table 6). The main effects of each independent variable on the unit density of the extrudates are presented in Table 4. For some of the response variables, significant differences were detected. Increasing SBM content of the blend, screw speed and screw compression ratio had no significant impacts on the unit density values of the extrudates. Increasing DDGS from 20 to $30 \%$ and temperature profile from 120 to $140^{\circ} \mathrm{C}$ decreased the UD by $6.7 \%$ and $7.5 \%$, respectively. As the $\mathrm{L} / \mathrm{D}$ of the die was increased from 9 to 13 , unit density increased by $9.1 \%$. With increases in moisture content from 20 to $40 \%$, unit density of the extrudates increased by $16.0 \%$, while expansion ratio decreased significantly. As reported by other studies, unit density is related to expansion ratio, which in turn is affected by the moisture content of the feed blend (Ding et al., 2005; Fang \& Hanna, 2000), which was supported by our results.

Unit density quantifies the density of a single extrudate. In aquafeeds, unit density plays a key role in the floatability of the feeds. For many fish species, such as Nile tilapia, floatability is recommended since they tend to feed close to the water surface. Extrudates that sink to the bottom of the tank may not be eaten, and present potential feed loss and, overtime, contamination of the water. Additionally, floating feed can show how much feed is consumed by fish and indicate changes in feeding behavior.

\subsubsection{Expansion Ratio}

Generally, expansion ratio is inversely related to the unit density. In this study, expansion ratio only related to the radial expansion (neglecting longitudinal and volumetric expansion), whereas unit density includes expansion in all directions. Expansion ratio varied between 0.53 and 1.05 within the treatment effects (Table 6). Significant differences were detected for most of the response variables. Expansion ratio significantly decreased by $22.2 \%$ by increasing moisture content from 20 to $40 \%$, and by $10.5 \%$ by increasing temperature from 100 to $140^{\circ} \mathrm{C}$ (Table 4 ). This conforms to the changes for the unit density, which increased with higher moisture content and higher temperature settings. Temperature impacts the rheological characteristics of the dough inside the extruder and thus, expansion ratio (Meng et al., 2010). Higher moisture reduces the viscosity of the melt and can act as a plasticizer, decreasing expansion ratio. With increasing screw speed (100 to $200 \mathrm{rpm}$ ), expansion ratio increased by $13.1 \%$, and with higher die L/D ratio (5 to 13 ), ER increased significantly by $17.1 \%$. Expansion ratio increased with lower die diameter. These results are in agreement with Sokhey et al. (1997), who determined that extrudate radial 
expansion significantly decreased with increasing die diameter when extruding yellow corn grits and Meng et al. (2010) who observed that increased screw speed led to increased expansion ratio.

\subsubsection{Pellet Durability Index}

Extruded feed should be of high enough quality to survive transportation and storage without breaking or major crumbling. Pellet durability index is typically used to assess an extrudates' ability to withstand destructive forces. Pellet durability index ranged from 86.49 to $98.99 \%$ (Table 6). Regarding the main effects, a significant difference was observed when increasing blend MC level from 30 to $40 \%$; PDI increased by $3.3 \%$. Increasing the moisture content from 20 to $40 \%$, on the other hand, yielded an increase in PDI by $6.0 \%$ (Table 4). This behavior was affected by the composition of the blends, which were relatively low in starch and high in protein. Similar observations due to moisture were likewise made in previous studies (Chevanan et al., 2008, 2009). Protein plasticizes under heat and will act as a binder when exposed to shear forces, heat, and moisture. No significant changes occurred with increasing DDGS levels, however, as observed in previous studies (Ayadi et al., 2011a; 2011b). Significantly reduced PDI was observed at the lowest die L/D ratio. This could be ascribed to nearly no expansion of the extrudate for this die geometry.

\subsubsection{Water Absorption Index}

Water absorption index (WAI) represents the hydrophilic aspect of blend formulation, while WSI is considered a measure of hydrophobic behavior (Ravindran et al., 2011). Starch-based materials have the ability to absorb water when the starch granules are damaged (Colonna et al., 1989). In this case, formulations were primarily protein-based; the blends used in these studies had low starch contents, which is reflected in the low WAI values, ranging between 2.77 and $3.90 \%$ (Table 6). The low starch content of the blends also prevented drastic changes in WAI due to the independent variables. Increasing the DDGS content from 20 to $40 \%$ and SBM from 30 to $50 \%$, yielded a decrease in WAI by $9.5 \%$ and $7.9 \%$, respectively, while an increase in temperature from 100 to $140^{\circ} \mathrm{C}$ yielded an increase of $8.5 \%$. The increase in WAI by higher temperatures can be related to the destruction of the crystalline structure of the starch, which allows it to absorb more water. Other researchers made similar observations for WAI with increasing temperature and DDGS content, respectively, when extruding DDGS-based feeds (Chevanan et al., 2007a, 2007b; Kannadhason et al., 2009b; Shukla et al., 2005). Increases in DDGS and SBM reduced the WAI of the blends due to less available starch in the blend. The die geometry also had some effect on WAI: a higher L/D ratio (from 5 to 13 ) resulted in significant increase in WAI by $10.9 \%$. This might be due to the increased expansion and thus destruction of starch granules. Increasing the screw compression from 2:1 to $3: 1$ resulted in a significant increase in WAI by $7.2 \%$ (Table 4). This was related to a higher compression, which increased shear and frictional forces, and resulted in a greater starch granule destruction.

\subsubsection{Water Solubility Index}

Referring to Kirby et al. (1988), WSI is related to the macromolecular degradation of starch. It is a measure of soluble polysaccharides that are cleaved by degradation of the starch granules (Ding et al., 2005). Water solubility index ranged from 17.43 to $22.72 \%$ (Table 6). Regarding the main effects, only a few significant differences were detected (Table 4). Increasing the DDGS level from 20 to $40 \%$, SBM level from 30 to 50\%, and die L/D ratio from 5 to 13 , yielded a decrease in WSI by $5.2 \%, 2.8 \%$ and $5.0 \%$, respectively. Similar results for WSI with increases in DDGS level were observed in previous studies (Chevanan et al., 2007a; Kannadhason et al., 2010). Extrusion cooking denatures proteins and releases hydrophobic amino acids that reduce solubility in water (Camire, 1991). With increasing moisture content (20 to $40 \%)$ and temperature settings $\left(100\right.$ to $\left.140^{\circ} \mathrm{C}\right)$, WSI showed a curvilinear behavior. It increased by $3.5 \%$ when raising MC from 20 to $30 \%$, but it decreased significantly by $4.4 \%$ when MC increased from 30 to 30\%; similar observations with increasing MC were made by Rosentrater et al. (2009b).

\subsubsection{Water Stability}

The length of time an extrudate will float without dissolving in water will dictate availability of feed for fish, loss of nutrients, and potential water pollution. Maintaining cohesive extrudates, once they are placed in water, is crucial. Water stability varied between $7.83 \mathrm{~min}$ and $30 \mathrm{~min}$ for all treatment combinations (Table 6). Only some significant differences were detected for the main effects (Table 4). This can be ascribed to the high standard deviations. An increase in MC from 20 to $40 \%$ yielded an increase in WS by $57.5 \%$. As for PDI, this behavior can be related to better binding which was achieved with higher MC. Blends with lower MC were more expanded and absorbed water faster. With a higher die L/D ratio (from 5 to 13 ), WS decreased significantly, by $26.3 \%$.

\subsubsection{Color}

The values for brightness (Hunter L) of the extrudates varied from 35.55 to 49.40 (Table 6), whereas the brightness of the raw materials varied from 51.57 to 62.43 (Table III). For the main effects, significant differences for the raw 
blends occurred only with increasing moisture content; brightness decreased by $9.8 \%$ when increasing the moisture content from 20 to $40 \%$. The level of DDGS or SBM did not affect the brightness of the raw ingredients. For the extruded material, brightness decreased significantly by $13.0 \%$ with a higher die L/D ratio, but it was not really affected by any other factor.

For redness (Hunter a), values ranged between 3.63 and 6.45 for the raw material, and between 3.21 and 6.92 for the extrudates. Regarding the main effects, redness of the unprocessed blends showed significant differences only with higher DDGS levels and higher moisture content. Redness increased by $26.7 \%$ when raising the DDGS content from 20 to $30 \%$, and by $28.0 \%$ when increasing the moisture content from 20 to $30 \%$. Similar changes were observed for yellowness (Hunter b).The values for yellowness ranged between 18.59 and 21.43 for the raw blends, and between 9.60 and 16.56 for the extrudates (Table 3 and 6). Increasing the DDGS amounts of the raw blends from 20 to $40 \%$, yielded a significant increase of $7.8 \%$ for yellowness. Increasing the moisture content from 20 to $40 \%$ yielded an increase of $6.5 \%$ (Table 4 ). Treatment combination effects on extrudate brightness are shown Figure 2. The generated response surface indicated a downward trend for extrudate brightness value with increase in both raw blend moisture content and DDGS level. As shown in Figure 2, raw blend moisture content exerted a greater effect on extrudate brightness than DDGS level.

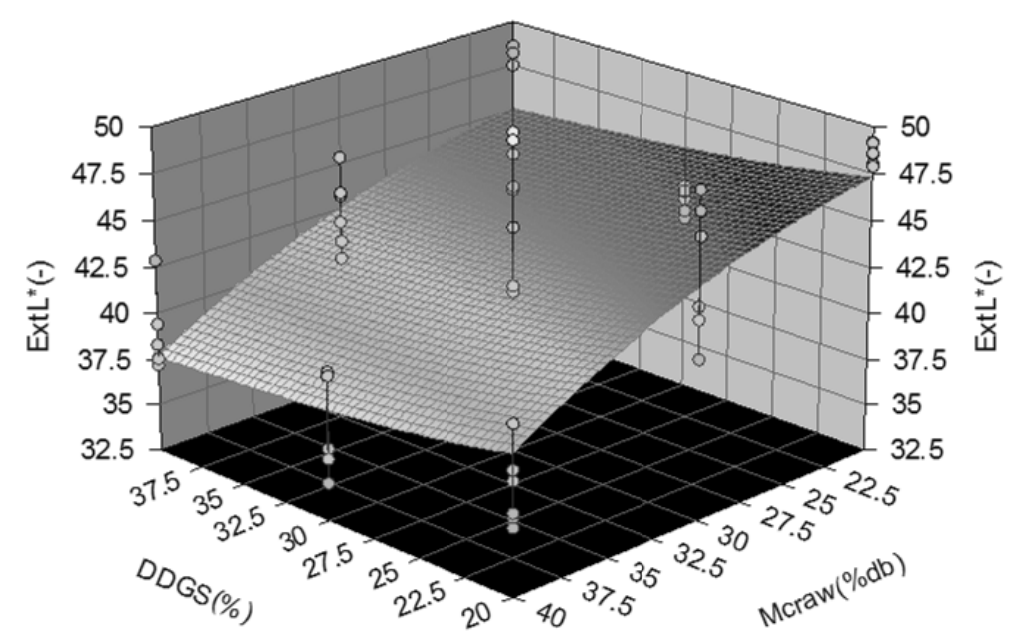

Figure 2. Treatment combination effects on extrudate brightness (Hunter $\mathrm{L}$ )

$\mathrm{L}_{\text {ext }}=43.88+90.59 / \mathrm{DDGS}(\mathrm{g} / \mathrm{kg})-1.33 * 10^{-4} * \mathrm{MC}_{\text {raw }}{ }^{3}(\mathrm{~g} / \mathrm{kg}) ;\left(\mathrm{R}^{2}=0.55, \mathrm{~F}=31.76\right)$.

\section{Conclusions}

This study was conducted to examine the effects of various levels of raw blend properties (such as DDGS, SBM, moisture), andprocessing conditions (screw speed, screw configuration ratio, die L/D ratio, and temperature profile) on extrusion processing conditions and extrudate physical properties. Raw moisture content and die dimensions significantly affected most of the extrudate physical properties. The pressure at the die decreased significantly with higher processing temperature, lower die L/D ratio, and higher screw compression, whereas the specific mechanical energy showed significant decrease only with higher DDGS content. The mass flow rate exhibited a significant increase with higher screw speed. Color and $\mathrm{a}_{\mathrm{w}}$ of the raw materials were only affected by raw moisture content and by DDGS level. Overall, it can be concluded that all independent variables had significant effects on certain dependent variables, while others did not. Changes of particular extruder and die settings, moisture content, and DDGS levels had significant effects on certain processing conditions and extrudate physical properties, and can be modified using various combinations thereof. Quantifying these relationships on a small scale is instructive, but this work needs to be done on either pilot or large scale processing equipment to truly understand these behaviors for commercial production of these types of diets. 


\section{Acknowledgements}

The authors thank the North Central Agricultural Research Laboratory, USDA-ARS, and the Agricultural Experiment Station, South Dakota State University, Brookings, South Dakota, for funding, facilities, equipment and supplies.

\section{References}

AACC. (2000). Method 44-19, moisture-air oven method, drying at $135^{\circ} \mathrm{C}$. AACC Approved Methods (10th ed.). St. Paul, MN: American Association of Cereal Chemists.

Akdogan, H. (1996). Pressure, torque, and energy responses of a twin screw extruder at high moisture contents. Food Research International, 29(5-6), 423-429. http://dx.doi.org/10.1016/S0963-9969(96)00036-1

Ali, Y., Hanna, M. A., \& Chinnaswamy, R. (1996). Expansion characteristics of extruded corn grits. Lebensmittel-Wissenschaft und-Technologie, 29(8), 702-707.

Anderson, R. A., Conway, H. F., Pfeifer, V. F., \& Griffin, L. E. (1969). Gelatinization of corn grits by rolland extrusion cooking. Cereal Science Today, 14, 4-7.

ASAE. (2004). Engineering Standards, Practices and Data. ASABE, St. Joseph, MI.

ATA. (2010). American Tilapia Association. Retrieved November 11, 2011, from http://ag.arizona.edu/azaqua/ata.html

Ayadi, F. Y., Muthukumarappan, K., Rosentrater, K. A., \& Brown, M. L. (2011a). Single-screw extrusion processing of distillers dried grains with solubles (DDGS)-based yellow perch (Perca flavescens) feeds. Cereal Chemistry, 88(2), 179-188. http://dx.doi.org/10.1094/CCHEM-08-10-0118

Ayadi, F. Y., Muthukumarappan, K., Rosentrater, K. A., \& Brown, M. L. (2011b). Twin-screw extrusion processing of rainbow trout (Oncorhynchus mykiss) feeds using various levels of corn-based distillers dried $\begin{array}{lllll}\text { grains with solubles (DDGS). Cereal } & \text { Chemistry, 88(4), }\end{array}$ http://dx.doi.org/10.1094/CCHEM-08-10-0120

Ayadi, F. Y., Rosentrater, K. A., \& Muthukumarappan, K. (2010d). A review of alternative protein sources in aquaculture feeds. American Society of Agricultural and Biological Engineers, 20-23 June 2010, Pittsburgh, Pennsylvania.

Ayadi, F. Y., Rosentrater, K. A., Muthukumarappan, K., \& Brown, M. L. (2011c). Twin-screw extrusion processing of distillers dried grains with solubles (DDGS)-based yellow perch (Perca flavescens) feeds. Food Bioprocess Technol. http://dx.doi.org/10.1007/s11947-011-0535-5

Bahnasawy, M. H. (2009). Effect of dietary protein levels on growth performance and body composition of monosex Nile tilapia, Oreochromis niloticus L. reared in fertilized tanks. Pakistan Journal of Nutrition, 8(5), 674-678. http://dx.doi.org/10.3923/pjn.2009.674.678

Bureau, D. P., Harris, A. M., Bevan, D. J., Simmons, L. A., Azevedo, P. A., \& Cho, C. Y. (2000). Feather meals and meat and bone meals from different origins as protein sources in rainbow trout (Oncorhynchus mykiss) diets. Aquaculture, 181(3-4), 281-291. http://dx.doi.org/10.1016/S0044-8486(99)00232-X

Camire, M. E. (1991). Protein functionality modification by extrusion cooking. Journal of the American Oil Chemists' Society, 68(3), 200-205.

Cheng, Z. J., \& Hardy, R. W. (2004). Nutritional value of diets containing distiller's dried grain with solubles for rainbow trout, Oncorhynchus mykiss. Journal of Applied Aquaculture, 15(3-4), 101-113. http://dx.doi.org/10.1300/J028v15n03_08

Chevanan, N., Muthukumarappan, K., \& Rosentrater, K. A. (2007c). Neural network and regression modeling of extrusion processing parameters and properties of extrudates containing DDGS. Transactions of the ASABE, 50(5), 1765-1778.

Chevanan, N., Muthukumarappan, K., \& Rosentrater, K. A. (2009). Extrusion studies of aquaculture feed using distillers dried grains with solubles and whey. Food and Bioprocess Technology, 2(2), 177-185. http://dx.doi.org/10.1007/s11947-007-0036-8

Chevanan, N., Muthukumarappan, K., Rosentrater, K. A., \& Julson, J. L. (2007a). Effect of die dimensions on extrusion processing parameters and properties of DDGS-based aquaculture feeds. Cereal Chemistry, 84(4), 389-398. http://dx.doi.org/10.1094/CCHEM-84-4-0389 
Chevanan, N., Rosentrater, K. A., \& Muthukumarappan, K. (2007b). Twin-screw extrusion processing of feed blends containing distillers dried grains with solubles (DDGS). Cereal Chemistry, 84(5), 428-436. http://dx.doi.org/10.1094/CCHEM-84-5-0428

Chevanan, N., Rosentrater, K. A., \& Muthukumarappan, K. (2008). Effect of DDGS, moisture content, and screw speed on physical properties of extrudates in single-screw extrusion. Cereal Chemistry, 85(2), 132-139. http://dx.doi.org/10.1094/CCHEM-85-2-0132

Chevanan, N., Rosentrater, K. A., \& Muthukumarappan, K. (2010). Effects of processing conditions on single screw extrusion of feed ingredients containing DDGS. Food and Bioprocess Technology, 3(1), 111-120. http://dx.doi.org/10.1007/s11947-008-0065-y

Chirife, J., \& Del Buera, P. M. (1994). Water activity, glass transition and microbial stability in concentrated/semi moist food systems. Journal of Food Science, 59(5), 921-927. http://dx.doi.org/10.1111/j.1365-2621.1994.tb08159.x

Colonna, P., Tayeb, J., \& Mercier, C. (1989). Extrusion cooking of starch and starchy products. In L. H. Mercier (Ed.), Extrusion cooking. American Association of Cereal Chemists (pp. 247-318). MN: Inc., St. Paul.

Ding, Q. B., Ainsworth, P., Tucker, G., \& Marson, H. (2005). The effect of extrusion conditions on the physicochemical properties and sensory characteristics of rice-based expanded snacks. Journal of Food Engineering, 66(3), 283-289. http://dx.doi.org/10.1016/j.jfoodeng.2004.03.019

El-Sayed, A. F. M., \& Teshima, S. I. (1992). Protein and energy requirements of Nile tilapia, Oreochromis niloticus, fry. Aquaculture, 103(1), 55-63. http://dx.doi.org/10.1016/0044-8486(92)90278-S

Fang, Q., \& Hanna, M. A. (2000). Functional properties of polylactic acid starch-based loose-fill packaging foams. Cereal Chemistry, 77(6), 779-783. http://dx.doi.org/10.1094/CCHEM.2000.77.6.779

FAO. (2010). Species Fact Sheets. Fisheries and Aquaculture Department. Retrieved November 11, 2011 from http://www.fao.org/fishery/species/3217/en

Fitzsimmons, K. (2006). Prospect and potential for global production. In C. Lim, \& C. D. Webster (Eds.), Tilapia: Biology, Culture, and Nutrition (pp. 51). Binghamton, NY: The Haworth Press, Inc.

Furuya, W. M., Pezzato, L. E., Barros, M. M., Pezzato, A. C., Furuya, V. R. B., \& Miranda, E. C. (2004). Use of ideal protein concept for precision formulation of amino acid levels in fish-meal-free diets for juvenile Nile tilapia (Oreochromis niloticus L.). Aquaculture Research, 35(12), 1110-1116. http://dx.doi.org/10.1111/j.1365-2109.2004.01133.x

Ganjyal, G. M., \& Hanna, M. A. (2004). Effects of extruder die nozzle dimensions on expansion and micrographic characterization during extrusion of acetylated starch. Starch - Stärke, 56(3-4), 108-117. http://dx.doi.org/10.1002/star.200300200

Guy, R. (2001). Extrusion Cooking: Technologies and Applications (p 89). Cambridge, UK: Woodhead Publishing Limited and CRC Press LLC.

Hafedh, Y. S. (1999). Effects of dietary protein on growth and body composition of Nile tilapia, Oreochromis niloticus L. Aquaculture Research, 30(5), 385-393. http://dx.doi.org/10.1046/j.1365-2109.1999.00343.x

Harper, J. M. (1981). Extrusion of Foods. FL, USA: CRC Press Inc., Boca Raton.

Jones, D., Chinnaswamy, R., Tan, Y., \& Hanna, M. A. (2000). Physiochemical properties of ready-to-eat breakfast cereals. Cereal Foods World, 45(4), 164-168.

Kannadhason, S., Muthukumarappan, K., \& Rosentrater, K. A. (2009a). Effect of starch sources and protein content on extruded aquaculture feed containing DDGS. Food and Bioprocess Technology (in press). http://dx.doi.org/10.1007/s11947-008-0177-4

Kannadhason, S., Muthukumarappan, K., \& Rosentrater, K. A. (2009b). Effects of ingredients and extrusion parameters on aquafeeds containing DDGS and tapioca starch. Journal of Aquaculture Feed Science and Nutrition, 1(1), 6-21.

Kannadhason, S., Rosentrater, K. A., \& Muthukumarappan, K. (2010). Twin screw extrusion of DDGS-based aquaculture feeds. Journal of the World Aquaculture Society, 41(51), 1-15. http://dx.doi.org/10.1111/j.1749-7345.2009.00328.x

Kirby, A. R., Ollett, A. L., Parker, R., \& Smith, A. C. (1988). An experimental study of screw configuration effects in the twin-screw extrusion-cooking of maize grits. Journal of Food Engineering, 8(4), 247-272. http://dx.doi.org/10.1016/0260-8774(88)90016-7 
Launay, B., \& Lisch, J. M. (1983). Twin-screw extrusion cooking of starches: Flow behaviour of starch pastes, expansion and mechanical properties of extrudates. Journal of Food Engineering, 2(4), 259-280. http://dx.doi.org/10.1016/0260-8774(83)90015-8

Lim, C., Garcia, J. C., Yildirim-Aksoy, M., Klesius, P. H., Shoemaker, C. A., \& Evans, J. J. (2007). Growth response and resistance to Streptococcus iniae of Nile tilapia, Oreochromis niloticus, fed diets containing distiller's dried grains with solubles. Journal of the World Aquaculture Society, 38(2), 231-237. http://dx.doi.org/10.1111/j.1749-7345.2007.00093.x

Lim, C., Yildirim-Aksoy, M., \& Klesius, P. H. (2009). Growth response and resistance to Edwardsiella ictaluri of channel catfish, Ictalurus punctatus, fed diets containing distiller's dried grains with solubles. Journal of the World Aquaculture Society, 40(2), 182-193. http://dx.doi.org/10.1111/j.1749-7345.2009.00241.x

Lin, S., Huff, H. E., \& Hsieh, F. (2002). Extrusion process parameters, sensory characteristics, and structural properties of a high moisture soy protein meat analog. Journal of Food Science, 67(3), 1066-1072. http://dx.doi.org/10.1111/j.1365-2621.2002.tb09454.x

Lowe, J. A., \& Kershaw, S. J. (1995). Water activity-moisture content relationship as a predictive indicator for control of spoilage in commercial pet diet components. Animal Feed Science and Technology, 56(3-4), 187-194. http://dx.doi.org/10.1016/0377-8401(95)00833-0

Lu, Q. (1992). Dynamic analysis of process variables for a twin-screw food extruder. Lebensm. Wiss, Technol, 25, 261-270.

Meng, X., Threinen, D., Hansen, M., \& Driedger, D. (2010). Effects of extrusion conditions on system parameters and physical properties of a chickpea flour-based snack. Food Research International, 43(2), 650-658. http://dx.doi.org/10.1016/j.foodres.2009.07.016

Metts, L. S., Thompson, K. R., Xiong, Y., Kong, B., Webster, C. D., \& Brady, Y. (2007). Use of alfalfa hay, compared to feeding practical diets containing two protein levels, on growth, survival, body composition, and processing traits of Australian red claw crayfish, Cherax quadricarinatus, grown in ponds. Journal of the World Aquaculture Society, 38(2), 218-230. http://dx.doi.org/10.1111/j.1749-7345.2007.00091.x

Njiru, M., Okeyo-Owuor, J. B., Muchiri, M., \& Cowx, I. G. (2004). Shifts in the food of Nile tilapia, Oreochromis niloticus (L.) in Lake Victoria, Kenya. African Journal of Ecology, 42(3), 163-170. http://dx.doi.org/10.1111/j.1365-2028.2004.00503.x

Pezzato, L. E. (1999). The use of extruded feed in fish food. In Y. K. Chang, \& S. S. Wang (Eds.), Advances in Extrusion Technology: Aquaculture Animal Feeds and Foods. Lancaster, PA, USA: Technomic Publishing Company.

Ravindran, G., Carr, A., \& Hardacre, A. (2011). A comparative study of the effects of three galactomannans on the functionality of extruded pea-rice blends. Food Chemistry, 124(4), 1620-1626. http://dx.doi.org/10.1016/j.foodchem.2010.08.030

Rawles, S. D., Gaylord, T. G., McEntire, M. E., \& Freeman, D. W. (2009). Evaluation of poultry by-product meal in commercial diets for hybrid striped bass, morone chrysops $\phi \times$ Morone saxatilis $\hat{\gamma}$, in pond production. Journal of the World Aquaculture Society, 40(2), 141-156. http://dx.doi.org/10.1111/j.1749-7345.2009.00238.x

Rawles, S. D., Thompson, K. R., Brady, Y. J., Metts, L. S., Gannam, A. L., Twibell, R. G., \& Webster, C. D. (2010). A comparison of two faecal collection methods for protein and amino acid digestibility coefficients of menhaden fish meal and two grades of poultry by-product meals for market-size sunshine bass (Morone $\begin{array}{llll}\text { chrysops } \times M . & \text { saxatilis }) . & \text { Aquaculture } & \text { Nutrition, }\end{array}$ http://dx.doi.org/10.1111/j.1365-2095.2008.00643.x

Rogers, M. G. (1970). Rheological interpretation of Brabender plasti-corder (extruder head) data. Industrial and Engineering Chemistry, 9(1), 49-52. http://dx.doi.org/10.1021/i260033a009

Romana-Eguia, M. R. R., Ikeda, M., Basiao, Z. U., \& Taniguchi, N. (2004). Genetic diversity in farmed Asian Nile and red hybrid tilapia stocks evaluated from microsatellite and mitochondrial DNA analysis. Aquaculture, 236(1-4), 131-150. http://dx.doi.org/10.1016/j.aquaculture.2004.01.026

Rosentrater, K. A., Muthukumarappan, K., \& Kannadhason, S. (2009a). Effects of ingredients and extrusion parameters on aquafeeds containing DDGS and potato starch. Journal of Aquaculture Feed Science and Nutrition, 1(1), 22-38. 
Rosentrater, K. A., Muthukumarappan, K., \& Kannadhason, S. (2009b). Effects of ingredients and extrusion parameters on properties of aquafeeds containing DDGS and corn starch. Journal of Aquaculture Feed Science and Nutrition, 1(2), 44-60.

Rosentrater, K. A., Richard, T. L., Bern, C. J., \& Flores, R. A. (2005). Small scale extrusion of corn masa by-products. Cereal Chemistry, 82(4), 436-446. http://dx.doi.org/10.1094/CC-82-0436

SAS. (2004). Windows NT Version 9. 2600. SAS Institute, Inc. Cary, North Carolina, USA.

Schaeffer, T. W., Brown, M. L., \& Rosentrater, K. A. (2009). Performance characteristics of Nile tilapia (Oreochromis niloticus) fed diets containing graded levels of fuel-based distillers dried grains with solubles. Journal of Aquaculture Feed Science and Nutrition, 1(4), 78-83. http://dx.doi.org/10.3923/joafsnu.2009.78.83

Schaeffer, T. W., Brown, M. L., Rosentrater, K. A., \& Muthukumarappan, K. (2010). Utilization of diets containing graded levels of ethanol production co-products by Nile tilapia. Journal of Animal Physiology and Animal Nutrition, 94, e348-e354. http://dx.doi.org/10.1111/j.1439-0396.2010.01020.x

Senouci, A., \& Smith, A. C. (1988). An experimental study of food melt rheology - I. Shear viscosity using a slit die viscometer and a capillary rheometer. Rheologica Acta, 27(5), 546-554. http://dx.doi.org/10.1007/BF01329355

Shukla, C. Y., Muthukumarappan, K., \& Julson, J. L. (2005). Effect of single-screw extruder die temperature, amount of distillers' dried grains with solubles (DDGS), and initial moisture content on extrudates. Cereal Chemistry, 82(1), 34-37. http://dx.doi.org/10.1094/CC-82-0034

Singh, B., Sekhon, K. S., \& Singh, N. (2007). Effects of moisture, temperature and level of pea grits on extrusion behaviour and product characteristics of rice. Food Chemistry, 100(1), 198-202. http://dx.doi.org/10.1016/j.foodchem.2005.09.042

Sokhey, A. S., Ali, Y., \& Hanna, M. A. (1997). Effects of die dimensions on extruder performance. Journal of Food Engineering, 31(2), 251-261. http://dx.doi.org/10.1016/S0260-8774(96)00025-8

Steffens, W. (1994). Replacing fish meal with poultry by-product meal in diets for rainbow trout, Oncorhynchus mykiss. Aquaculture, 124(1-4), 27-34. http://dx.doi.org/10.1016/0044-8486(94)90351-4

Thompson, K. R., Rawles, S. D., Metts, L. S., Smith, R., Wimsatt, A., Gannam, A. L., .. Webster, C. D. (2008). Digestibility of dry matter, protein, lipid, and organic matter of two fish meals, two poultry by-product meals, soybean meal, and distiller's dried grains with solubles in partial diets for sunshine bass, Morone chrysops $X$ M. saxatilis. Journal of the World Aquaculture Society, 39(3), 352-363. http://dx.doi.org/10.1111/j.1749-7345.2008.00174.x

USDA. (1999). Practical procedures for grain handlers: Inspecting grain. Washington, D.C.: Grain Inspection, Packers, and Stockyards Administration, United States Department of Agriculture. Retrieved August 18, 2010 from http://archive.gipsa.usda.gov/pubs/primer.pdf

USDA. (2010). Byproduct feeds: Average wholesale price, bulk, specified markets. Feed Grains Data: Yearbook Tables. Washington, D.C.: USDA Agricultural Marketing Service. Retrieved November 11, 2011 from http://www.ers.usda.gov/Data/FeedGrains/Yearbook/FGYearbookTable16.pdf.

Wang, L., Chessari, C., \& Karpiel, E. (2001). Inferential control of product quality attributes-application to food cooking extrusion process. Journal of Process Control, 11(6), 621-636. http://dx.doi.org/10.1016/S0959-1524(00)00055-X

Webster, C. D., \& Lim, C. E. (2002). Preface. In C. D. Webster, C. E. Lim (Eds.), Nutrient Requirements and Feeding of Finfish for Aquaculture. Wallingford, Oxford, UK: CABI Publishing.

Webster, C. D., Tidwell, J. H., \& Goodgame, L. S. (1993). Growth, body composition, and organoleptic evaluation of channel catfish fed diets containing different percentages of distillers' grains with solubles. The Progressive Fish-Culturist, $\quad$ 55(2), 95-100. http://dx.doi.org/10.1577/1548-8640(1993)055<0095:GBCAOE >2.3.CO;2

Wu, V. W., Rosati, R. R., \& Brown, P. B. (1996). Effect of diets containing various levels of protein and ethanol coproducts from corn on growth of tilapia fry. Journal of Agricultural and Food Chemistry, 44(6), 1491-1493. 